\title{
REVIEW ARTICLE OPEN Crosstalk between circRNAs and the PI3K/AKT signaling pathway in cancer progression
}

\author{
Chen Xue ${ }^{1}$, Ganglei $\mathrm{Li}^{2}$, Juan $\mathrm{Lu}^{1}$ and Lanjuan $\mathrm{Li}^{1 凶}$
}

Circular RNAs (circRNAs), covalently closed noncoding RNAs, are widely expressed in eukaryotes and viruses. They can function by regulating target gene expression, linear RNA transcription and protein generation. The phosphoinositide 3-kinase (PI3K)/AKT signaling pathway plays key roles in many biological and cellular processes, such as cell proliferation, growth, invasion, migration, and angiogenesis. It also plays a pivotal role in cancer progression. Emerging data suggest that the circRNA/PI3K/AKT axis modulates the expression of cancer-associated genes and thus regulates tumor progression. Aberrant regulation of the expression of circRNAs in the circRNA/PI3K/AKT axis is significantly associated with clinicopathological characteristics and plays an important role in the regulation of biological functions. In this review, we summarized the expression and biological functions of PI3K-AKTrelated circRNAs in vitro and in vivo and assessed their associations with clinicopathological characteristics. We also further discussed the important role of circRNAs in the diagnosis, prognostication, and treatment of cancers.

Signal Transduction and Targeted Therapy (2021)6:400

; https://doi.org/10.1038/s41392-021-00788-w

\section{INTRODUCTION}

The complexity of cancer and the variability of its clinical features are derived from its complex etiology, involving DNA, RNA, protein, and other factors. ${ }^{1-3}$ Cancer has become an important public health concern affecting people's lives. ${ }^{4-6}$ In the past 10 years, the number of studies on cancer has increased rapidly, providing many novel clues for the treatment of cancer. 7,8 The emergence of targeted therapy and immunotherapy has greatly improved the survival rate of cancer patients. ${ }^{9,10}$ However, cancer treatment remains a major scientific challenge.

Circular RNAs (circRNAs), a newly discovered type of noncoding RNA, have a covalently closed structure and high stability. ${ }^{11-13}$ CircRNAs are mainly formed by pre-mRNA a back-splicing and are widely expressed in eukaryotes and viruses. ${ }^{14,15}$ The regulatory role of circRNAs in physiological processes is still not very clear. ${ }^{16}$ However, accumulating evidence indicates that circRNAs are significantly associated with many diseases and play an important role in the occurrence and development of cancer. A common circRNA-mediated mechanism is that circRNAs act as competitive endogenous RNAs (ceRNAs) of microRNAs (miRNAs) in tumor progression. Circ101237 facilitates the expression of MAPK1 to suppress tumor progression by sponging miR-490-3p in non-small cell lung cancer (NSCLC). ${ }^{17}$ CircRNA also regulates cancer development and progression by interacting with protein. CircRNA clARS suppresses cell autophagy via binding with RBP ALKBH5. ${ }^{18}$

Phosphoinositide 3-kinase ( $\mathrm{PI} 3 \mathrm{~K})$, a member of the lipid kinase family, is an important regulator of signaling and intracellular vesicular trafficking. ${ }^{19}$ Several studies have found that the PI3K/ AKT pathway is aberrantly activated in cancer ${ }^{20-22}$ and controls core cellular functions, such as proliferation and survival. ${ }^{23,24}$ The $\mathrm{PI} 3 \mathrm{~K} / \mathrm{AKT}$ pathway plays a pivotal role in the progression of cancer. Clinical trials targeting PI3K have also attracted increasing attention. ${ }^{25,26}$ Emerging evidence suggests that circRNAs interact with the PI3K/AKT pathway to regulate cancer progression. Importantly, circRNAs related to the PI3K/AKT pathway have become potential targets in the treatment of cancer. In this review, we summarized the current studies of the role of crosstalk between circRNAs and the PI3K/AKT pathway in the initiation and progression of cancer (Fig. 1). We also presented the clinical applications of PI3K/AKT-related circRNAs in patients with cancer.

\section{THE PI3K/AKT SIGNALING PATHWAY IN TUMORIGENESIS} PI3K

Phosphoinositide 3-kinase (PI3K), a member of the lipid kinase family, ${ }^{27,28}$ was first identified 3 decades ago. ${ }^{29}$ It can be divided into 3 types (class I-III) in mammals. ${ }^{19,30,31}$ Class I PI3Ks have gained much attention in the cancer-related field. PI3K is composed of one catalytic ( $\mathrm{p} 110)$ domain and one regulatory (p85) domain. ${ }^{32,33}$ p85, which contains the Src homology 2 (SH2) and $\mathrm{SH} 3$ protein-binding domains, ${ }^{34,35}$ can interact with target proteins with corresponding binding sites. The activation of PI3K mainly involves the binding of the substrate near the inner side of the plasma membrane. ${ }^{36,37} \mathrm{PI} 3 \mathrm{~K}$ can be activated in two ways. One is that $\mathrm{PI} 3 \mathrm{~K}$ interacts with connexin or growth factor receptors with phosphorylated tyrosine residues, and then induces a conformational change of dimer. ${ }^{38-40}$ It also can be activated by the direct binding of 110 and Ras. ${ }^{41-43}$

\footnotetext{
${ }^{1}$ State Key Laboratory for Diagnosis and Treatment of Infectious Diseases, National Clinical Research Center for Infectious Diseases, Collaborative Innovation Center for Diagnosis and Treatment of Infectious Diseases, The First Affiliated Hospital, College of Medicine, Zhejiang University, Hangzhou 310003, China and ${ }^{2}$ Department of Neurosurgery, The First Affiliated Hospital, College of Medicine, Zhejiang University, Hangzhou 310003, China

Correspondence: Lanjuan Li (ljli@zju.edu.cn)

These authors contributed equally: Chen Xue, Ganglei Li, Juan Lu
}

Received: 21 June 2021 Revised: 30 September 2021 Accepted: 8 October 2021

Published online: 24 November 2021 
PI3K can be activated by multiple growth factors and signaling complexes, such as G-protein coupled receptors, B-cell receptors, vascular endothelial growth factor (VEGF), fibroblast growth factor (FGF), insulin and receptor tyrosine kinases (RTKs) (Fig. 2). ${ }^{20,44-48}$ These factors induce autophosphorylation through the activation of RTKs and then activate $\mathrm{PI} 3 \mathrm{~K}^{49}$ The $\mathrm{p} 85$ subunit provides docking sites for autophosphorylation. In some cases, this process is mediated by the recruitment of adapter proteins. For example, the insulin receptor activates PI3K via insulin receptor substrate- 1 (IRS-1). ${ }^{50,51}$ Activated PI3K increases the conversion of PIP2 to PIP3,

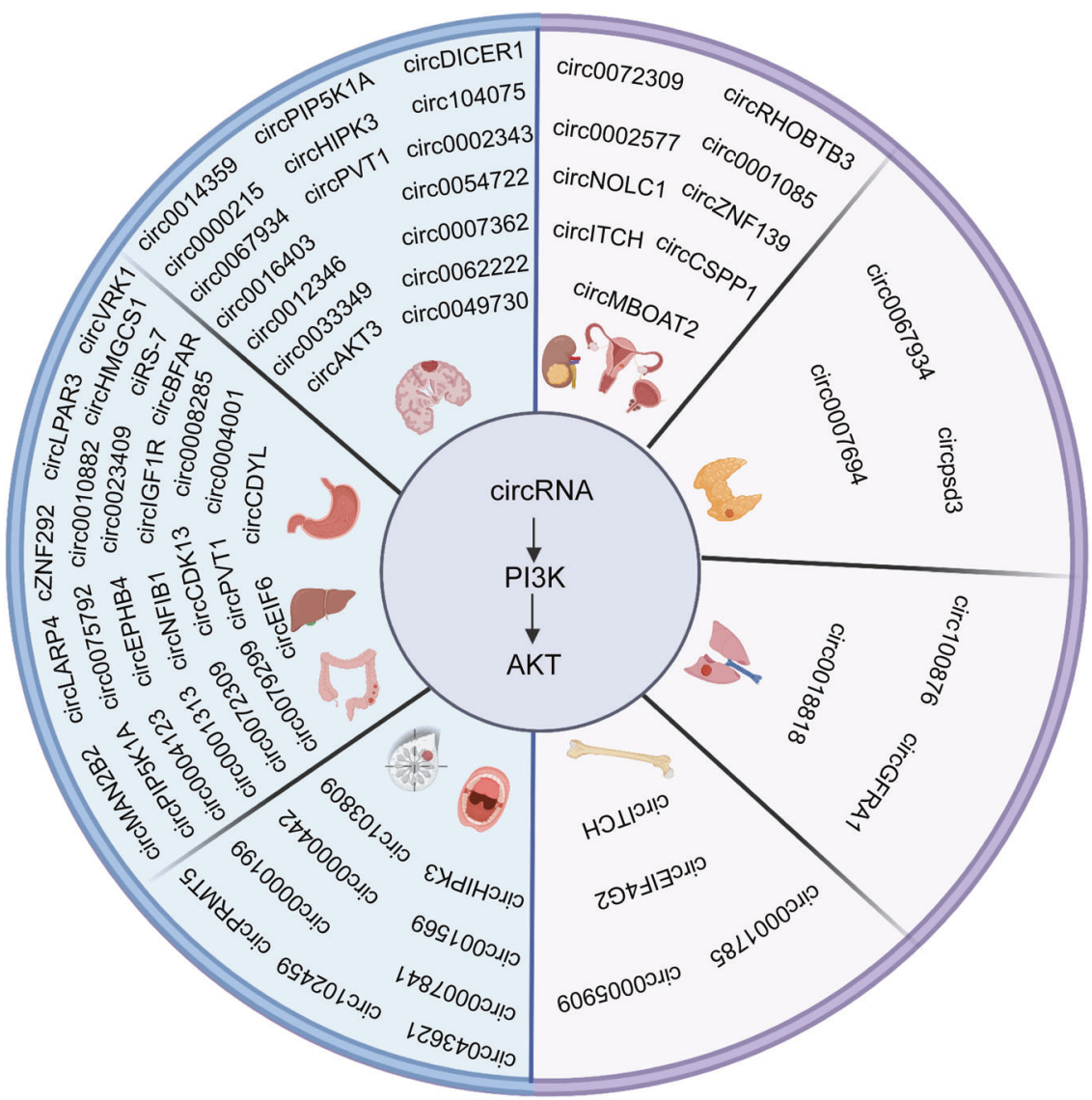

Fig. 1 CircRNAs interact with the PI3K/AKT pathway to regulate cancer progression. Image created with BioRender (https://biorender.com/)

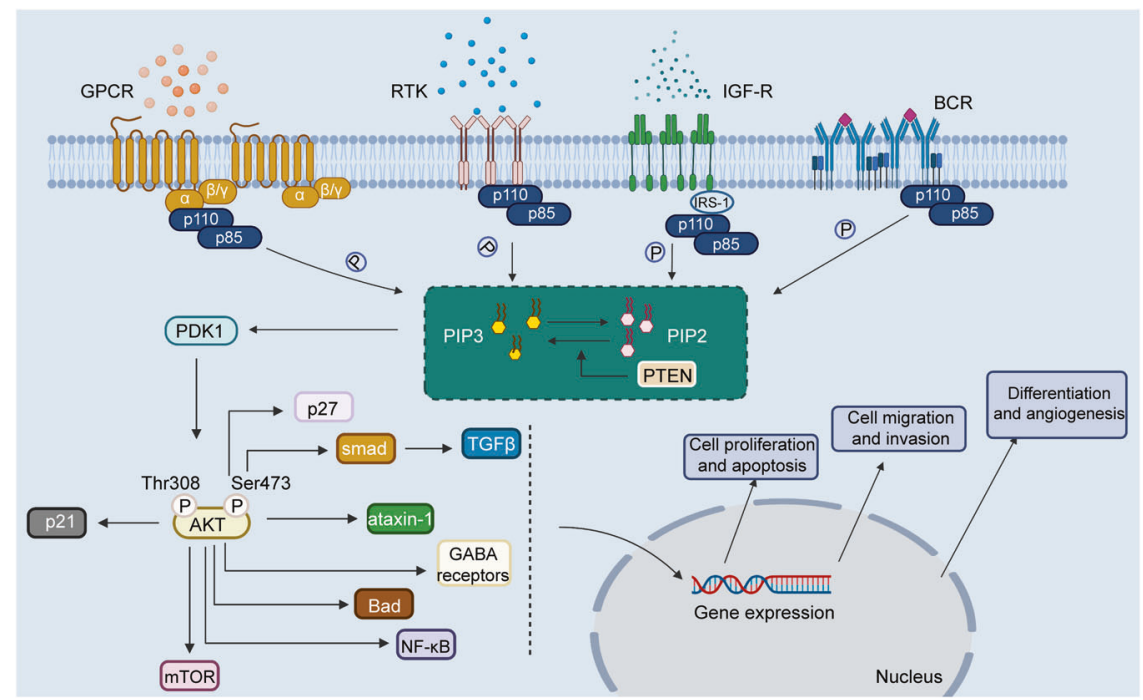

Fig. 2 The activation process of PI3K/AKT signaling pathway. PI3K, composed of one catalytic ( $\mathrm{p} 110)$ domain and one regulatory (p85), can be activated by G-protein coupled receptor, RTK, IGF-R, and B-cell receptor. Activated PI3K facilitates the conversion of PIP2 to PIP3. PIP3 activates PDK1, and then PDK1 phosphorylates AKT at Thr308. AKT can be also phosphorylated, and activated by PDK2 at Ser473. Activated AKT can regulate mangy cellular biological functions by interacting with numerous downstream signaling molecules, such as p21, p27, TGF $\beta$, ataxin-1, GABA receptors, Bad, NF- $\mathrm{kB}$, and mTOR. Image created with BioRender (https://biorender.com/) 
which activates PDK1 and AKT. ${ }^{52,53}$ However, AKT is not the only target molecule of PI3K. PI3K regulates multiple signaling pathways by interacting with BTK, PDK1, and Rac. ${ }^{54}$

AKT

AKT, also called protein kinase B (PKB), ${ }^{55,56}$ is the cellular homolog of the oncogene v-Akt. AKT is a serine/threonine kinase that belongs to the AGC kinase family. ${ }^{57-59}$ There are three different AKT isoforms (AKT1, AKT2, and AKT3), which are widely expressed in most human tissues. ${ }^{60-62}$ AKT can link the interaction between receptors and PI3K to cellular anabolic pathways. AKT acts as a central regulator of cellular metabolism downstream of insulin signaling that is responsible for the regulation of glucose metabolism. $^{63,64}$ In vivo experiments support that AKT2 plays a key role in the regulation of glucose metabolism. ${ }^{6,66}$ Researchers have found that germline mutations of AKT occur during the tumorigenesis and progression of some cancer. ${ }^{67,68}$

AKT plays a key role in multiple cellular processes, such as cell survival, proliferation, migration, apoptosis, and angiogenesis. ${ }^{69-72}$ AKT prevents TSC1/TSC2 complex formation and activates mTOR pathway, thereby regulating cell growth. ${ }^{73-75}$ It also regulates the expression of cyclin D1 and p53 to affect the cell cycle or the proliferation of various cell types through interacting with CDK inhibitors including p21 and p27. ${ }^{76}$ AKT boosts cell survival via inactivating the pro-apoptotic factors Bad and the transcription factor of the Forkhead (FKHR) family. ${ }^{77}$ The expression levels of GABA receptors and ataxin- 1 are also regulated by AKT. ${ }^{78,79}$ Some studies observed that AKT regulates the TGF $\beta$ signaling pathway by binding with Smad. ${ }^{80}$ The present findings show that AKT is an important target for the treatment of cancer, diabetes, stroke, and neurodegenerative diseases. ${ }^{81-83}$

The activation of PI3K/AKT pathway

The PI3K/AKT signaling pathway plays key role in many biological and cellular functions. ${ }^{84,85}$ We have already elaborated on the activation of $\mathrm{PI} 3 \mathrm{~K}$ when introducing $\mathrm{PI} 3 \mathrm{~K}$. The inositol ring of $\mathrm{PI}$ has five potential phosphorylation sites. PI3K activation could catalyze the phosphorylation of phosphatidylinositol (PI) at the $3^{\prime}$ position of the inositol ring. ${ }^{86}$ The phosphorylated products have a critical influence on cellular functions. PIP3 could enhance cell migration, ${ }^{87}$ and PI 3,4-bisphosphate regulates $B$ cell activation and insulin sensitivity. ${ }^{88}$ AKT and PDK1, which contain PH domains can bind to PIP3. PIP3 activates PDK1, ${ }^{89}$ and then PDK1 phosphorylates AKT at Thr308. ${ }^{90,91}$ AKT can be also phosphorylated and activated by PDK2 at Ser473. ${ }^{92,93}$ Activated AKT regulates cell proliferation, differentiation, migration, and apoptosis by activating or inhibiting downstream target proteins, such as Bad ${ }^{94}$ Caspase $9,{ }^{95}$ NF-KB, ${ }^{96,97}$ GSK-3, ${ }^{98}$ FKHR, ${ }^{99,100}$ p21, ${ }^{101}$ p53 ${ }^{102}$ and FOXO1. ${ }^{103,104}$ Aberrant activation of PI3K/AKT pathway has been found in a variety of cancers, ${ }^{105}$ such as lung cancer, ${ }^{106}$ esophageal cancer, ${ }^{107}$ gastric cancer, ${ }^{108}$ breast cancer, ${ }^{109}$ laryngeal cancer, ${ }^{110}$ gallbladder cancer, ${ }^{111}$ and prostate cancer. ${ }^{112}$

PTEN is a widely mutated tumor suppressor gene that inhibits the oncogenic PI3K/AKT pathway. ${ }^{113-115}$ PTEN antagonizes the PI3K/Akt pathway by dephosphorylating PIP3 to PIP2, ${ }^{116,117}$ then induces changes in a variety of cellular biological functions. ${ }^{18,119}$ Carboxyl-terminal modulator protein (CTMP) could block the transmission of downstream signaling pathways by inhibiting AKT phosphorylation. ${ }^{120,121}$ PP2A has been found to dephosphorylate AKT-Thr308 and AKT-Ser473 to inhibit the activation of AKT. ${ }^{12,123}$

\section{CIRCRNAS AND CANCER}

CircRNAs were initially found in RNA viruses at the end of the 20th century and were considered transcriptional background noise. ${ }^{124-126}$ With the application of high-throughput RNA sequencing and bioinformatics approaches, circRNAs have attracted much attention from researchers. ${ }^{13,127,128}$ CircRNAs, covalently closed noncoding RNAs, are widely expressed in eukaryotes and viruses. ${ }^{11,129-131}$ Linear pre-mRNAs generate circRNAs through exon skipping or back-splicing events. ${ }^{132,133}$ The circular form of circRNAs protects them from degradation by exonucleases, causing them to show greater stability. ${ }^{11,12}$ CircRNAs can function by regulating target gene expression, linear RNA transcription, and protein generation. ${ }^{13,134,135}$ Moreover, circRNAs are involved in the occurrence and development of several cancers. ${ }^{129,136-139}$ Different circRNAs play distinct roles in diverse cancer types. The circRNA CSMARCA5 has tumorsuppressive properties in the progression of hepatocellular carcinoma. ${ }^{136}$ However, circMAPK4 suppresses cell apoptosis by regulating specific pathways in gliomas. ${ }^{140}$

There are mainly four mechanisms by which circRNAs can act in cancer progression: miRNA sponging, protein binding, regulation of gene transcription, and regulation of protein translation. CircRNAs function as natural miRNA sponges that regulate miRNA activity. ${ }^{141-143}$ miRNAs are essential players in almost all carcinogenic processes. ${ }^{144-146}$ Increasing evidence suggests that circRNAs modulate cancer progression by regulating the expression of miRNA targets. ${ }^{147-151}$ For example, cTFRC facilitates tumor progression by sponging miR-107 in bladder carcinoma. ${ }^{152}$ In addition, circRNAs regulate cancer development and progression by directly modifying the transcription of related genes. Zhang et al. ${ }^{153}$ reported a novel class of intron-derived circRNAs that is widely distributed throughout the nucleus. Intron-derived circRNAs can interact with RNA polymerase II to enhance the transcription of its target genes. ${ }^{154,155}$ CircRNAs could also act as protein decoys, and regulate RNA-binding proteins (RBPs) activity by combining with RBPs. ${ }^{156,157}$ The expression of circZKSCAN1 attenuates HCC cell stemness by targeting RBP fragile $X$ mental retardation protein. ${ }^{158}$ Moreover, some circRNAs containing the AUG start codon and IRES can control gene expression at the translational level. ${ }^{159,160}$ However, this effect has not yet been fully elucidated in cancer.

\section{THE CIRCRNA/PI3K/AKT AXIS IN CANCER}

CircRNA plays a critical role in the initiation and development of human cancer. ${ }^{161-165}$ The studies on circRNA are changing our view of cancer genesis, progression, and treatment. ${ }^{166,167}$ CircRNAs alone may be insufficient for driving cancer progression. Similarly, traditional signaling pathways or signaling molecules alone may also be ineffective. Interestingly, studies have found that circRNAs are often interrelated with the PI3K/AKT signaling pathway. The PI3K/AKT signaling pathway plays key roles in many biological and cellular functions, such as cell proliferation, growth, invasion, migration, and angiogenesis. ${ }^{85,168}$ It also plays a pivotal role in the progression of cancer. ${ }^{27,169,170}$ Recently, a great deal of research regarding the interaction of circRNA and PI3K/AKT signaling pathways has attracted significant research interest. CircRNAs regulate cellular functions and control the occurrence and development of cancer via interactions with the PI3K/AKT pathway. Based on the current study, the mechanism/pattern of interaction between circRNA and PI3K/AKT pathway is primarily the ceRNA mechanism, which involves the activation or repression of downstream pathways by sponging miRNA. Research on the circRNA/PI3K/AKT axis is still in its infancy. With the deepening of research about the structure and function of circRNAs, the mechanism will add clarity regarding the circRNA/PI3K/AKT axis.

\section{CLINICAL FEATURES AND CELL BIOLOGICAL FUNCTIONS RELATED TO THE CIRCRNA/PI3K/AKT AXIS}

A large number of circRNAs have been found to be involved in the $\mathrm{PI3K} / \mathrm{AKT}$ signaling pathway. The circRNA/PI3K/AKT axis modulates the expression of cancer-associated genes and thus regulates tumor progression. The circRNA/PI3K/AKT axis plays important role 


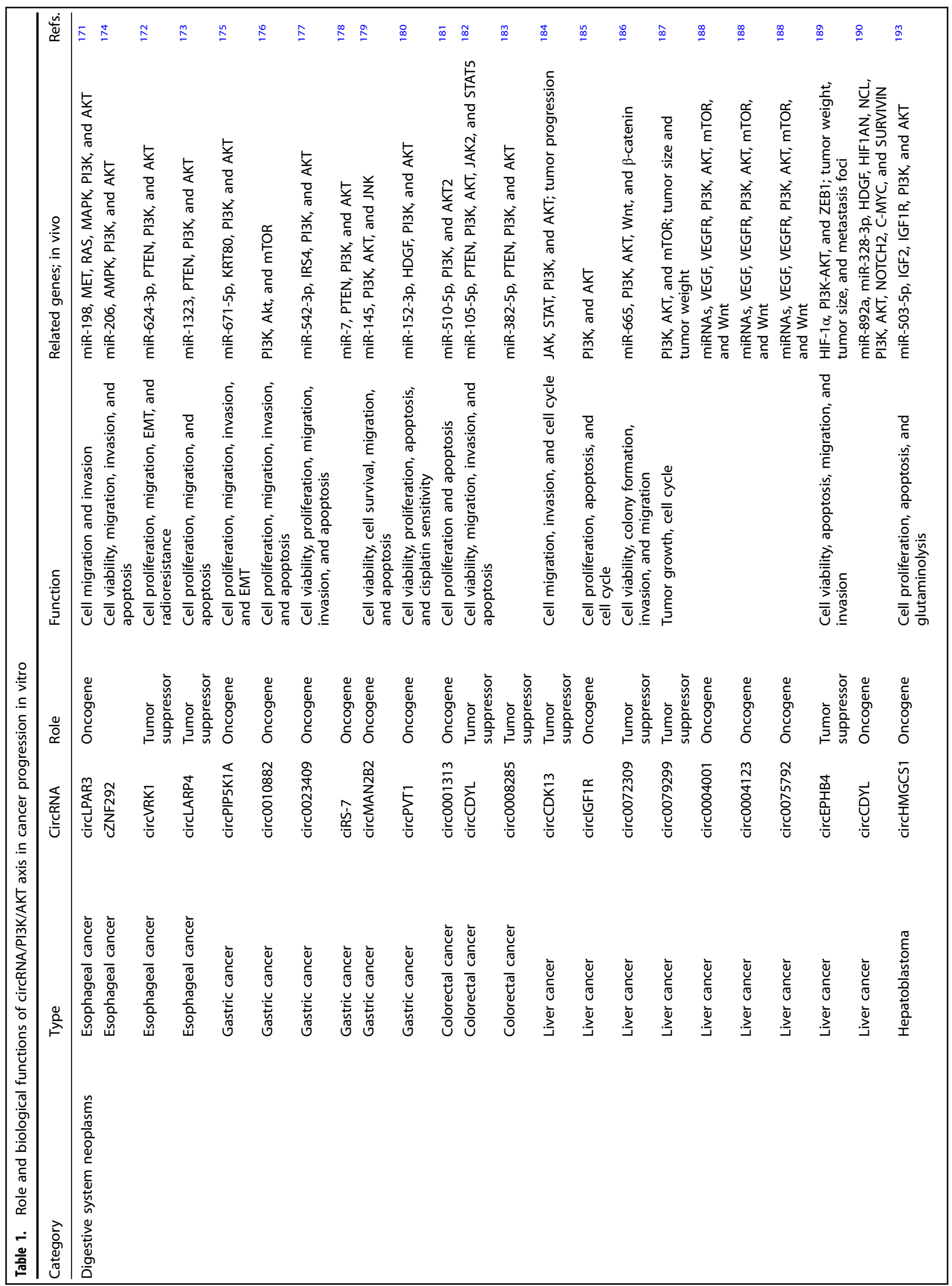




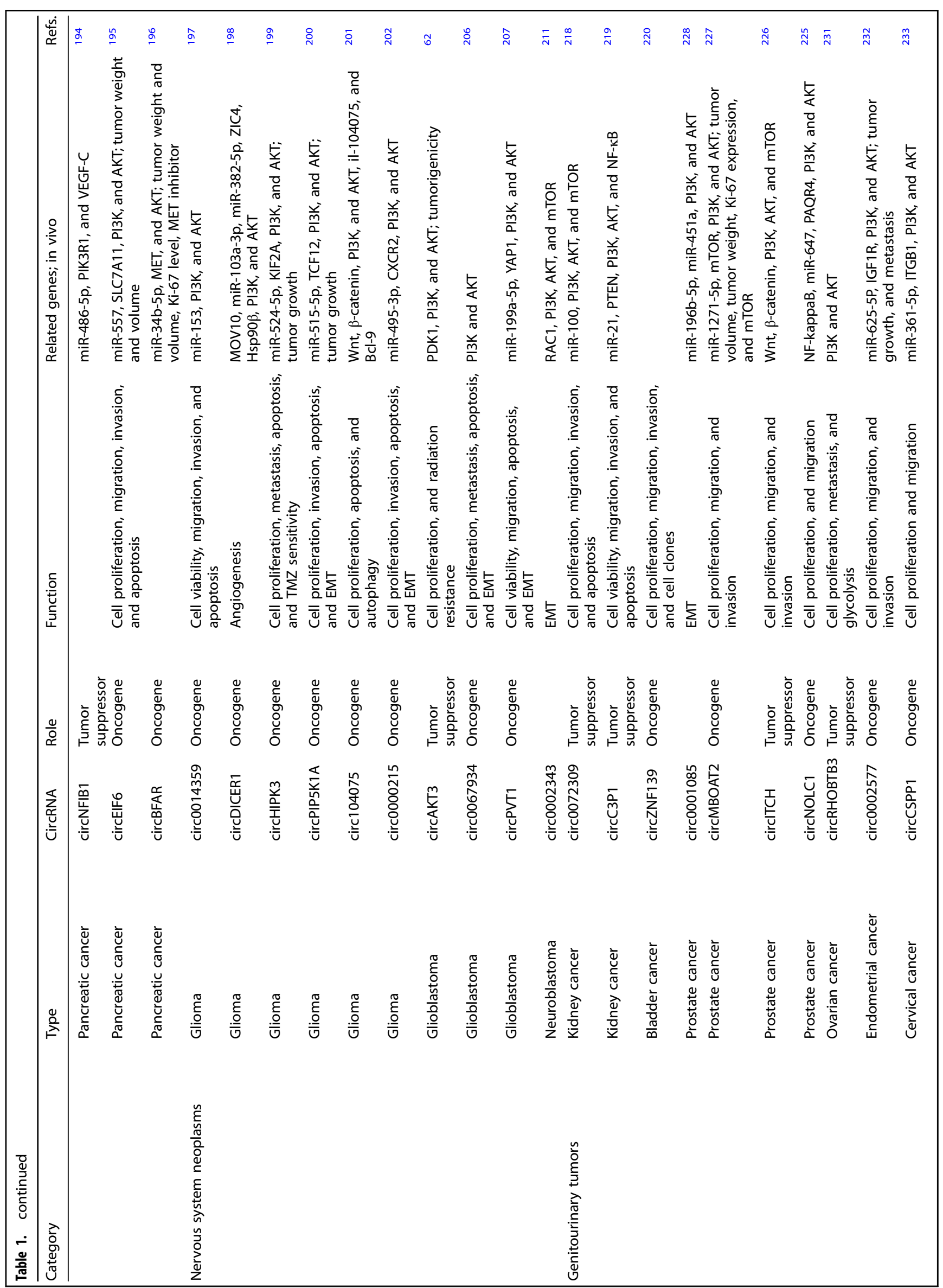




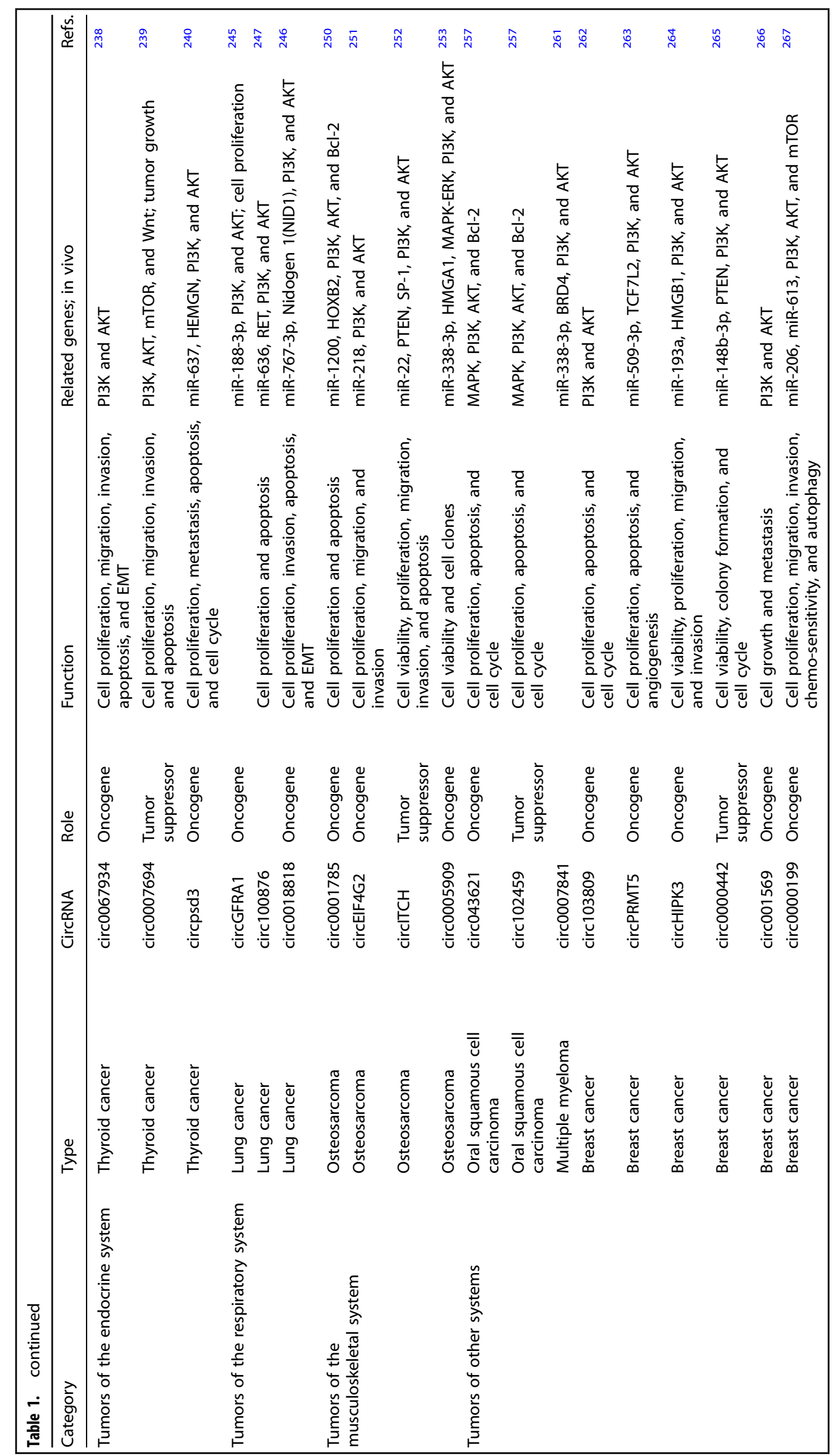


Table 2. Relationship between circRNA/PI3K/AKT axis and clinical features in cancer

\begin{tabular}{|c|c|c|c|c|}
\hline Cancer type & CircRNA & Expression & Related features & Refs. \\
\hline Liver cancer & circlGF1R & Upregulated & Tumor size & 185 \\
\hline Liver cancer & $\begin{array}{l}\text { circ0004001, circ0004123, and } \\
\text { circ0075792 }\end{array}$ & Upregulated & TNM stage, and tumor size & 188 \\
\hline Thyroid cancer & circ0067934 & Upregulated & Survival period and AJCC stage & 238 \\
\hline Glioblastoma & $\operatorname{circ0067934}$ & Upregulated & Disease-free survival and overall survival & 206 \\
\hline Colorectal cancer & circ0008285 & Downregulated & Lymph node metastasis, TNM stage, and tumor size & 183 \\
\hline Oral squamous cell carcinoma & circ043621 & Upregulated & $\begin{array}{l}\text { Clinical stage, lymph node metastasis, and } \\
\text { differentiation degree }\end{array}$ & 257 \\
\hline Oral squamous cell carcinoma & circ102459 & Downregulated & $\begin{array}{l}\text { Clinical stage, lymph node metastasis, and } \\
\text { differentiation degree }\end{array}$ & 257 \\
\hline Breast cancer & circ001569 & Upregulated & $\begin{array}{l}\text { Lymph node metastasis, pathological stage, and overall } \\
\text { survival }\end{array}$ & 266 \\
\hline Breast cancer & circ0000199 & Upregulated & Tumor size, TNM stage, ki-67 level, and 3-year survival & 267 \\
\hline Esophageal cancer & circLPAR3 & Upregulated & Lymph node metastasis and TNM stage & 171 \\
\hline Esophageal cancer & circVRK1 & Downregulated & Overall survival & 172 \\
\hline Gastric cancer & $\operatorname{circ0010882}$ & Upregulated & Tumor size, histological grade, and overall survival & 176 \\
\hline Gastric cancer & $\operatorname{circ0023409}$ & Upregulated & Tumor size, histological grade, and lymph nodes metastasis & 177 \\
\hline Gastric cancer & ciRS-7 & Upregulated & Overall survival & 178 \\
\hline Pancreatic cancer & circNFIB1 & Downregulated & Lymph node metastasis & 194 \\
\hline Pancreatic cancer & circBFAR & Upregulated & TNM stage, overall survival, and disease-free survival & 196 \\
\hline
\end{tabular}

in the initiation and progression of several types of cancer. Current studies may lay the foundation for further research on the mechanisms of cancer progression and provide insights into circRNA-based clinical applications. In this section, we will summarize the expression, biological functions in vitro (Table 1), and associations with clinicopathological characteristics of circRNAs related to the PI3K/AKT signaling pathway (Table 2).

\section{DIGESTIVE SYSTEM NEOPLASMS}

Esophageal cancer

The expression of circVRK1 and circLARP4 is significantly downregulated and circLPAR3 levels are increased in esophageal squamous cell carcinoma (ESCC). ${ }^{171-173}$ Low circVRK1 expression predicts poor overall survival in patients with ESCC. ${ }^{172}$ Elevated circLPAR3 levels are markedly associated with lymph node metastasis (LNM) and advanced TNM stage. ${ }^{171}$ In addition, researchers have also observed alterations in biological functions of the circRNA/PI3K/AKT axis by in vitro functional assays. Silencing of the circRNA cZNF292 inhibits the activity of tumor cells and promotes cell apoptosis in ESCC. ${ }^{174}$ Upregulation of circVRK1 suppresses cell proliferation, increases the radiosensitivity of ESCC cells, and attenuates epithelial-mesenchymal transition (EMT). ${ }^{172}$ CircLARP4 inhibits cell apoptosis and promotes cell proliferation in ESCC. ${ }^{173}$ Furthermore, cZNF292, circVRK1, and circLARP4 all inhibit ESCC cell migration. Contrary to the aforementioned investigations, circLPAR3 functions as a tumor oncogene and enhances the malignant phenotype of ESCC tumors. ${ }^{171}$ Mechanistically, circLPAR3 increases the expression of the MET gene to enhance the RAS/MAPK and PI3K/Akt pathways by sponging miR-198 in ESCC. Knockdown of cZNF292 induces inactivation of the PI3K/AKT pathway and upregulation of AMPK signaling to exert effects in ESCC. ${ }^{174}$ CircVRK1 functions as a tumor suppressor gene by upregulating PTEN and inhibiting the PI3K/ AKT axis. ${ }^{172}$ Similarly, circLARP4 promotes the expression of PTEN and inactivates the PI3K/AKT pathway to suppress the progression of ESCC. ${ }^{173}$

\section{Gastric cancer}

PI3K/AKT pathway-related circRNAs (circPIP5K1A, circ0010882, circ0023409, ciRS-7, circMAN2B2, and circPVT1) are all obviously upregulated in gastric cancer. ${ }^{175-180}$ The levels of circ0010882 and circ0023409 are positively associated with tumor size and histological grade in gastric cancer patients. ${ }^{176,177}$ In addition, higher expression of circ0010882 or ciRS-7 is associated with shorter overall survival. Circ0023409 promotes LNM in gastric cancer. In terms of biological function, increased circPIP5K1A, circ0010882, and circ0023409 expression reduces gastric cancer cell proliferation, migration, and invasion. ${ }^{175-177}$ High expression of circPVT1 may enhance the sensitivity of gastric cancer cells to cisplatin (DDP). ${ }^{180}$ We also found that circMAN2B2 upregulates cell viability and the surviving cell fraction by cell transfection experiments. ${ }^{179}$ Silencing of circ0010882 attenuated gastric cancer cell growth and motility in vitro. ${ }^{176}$ In terms of the mechanism, circPIP5K1A sponges miR-671-5p to facilitate tumor progression by upregulating the KRT80 and PI3K/AKT pathways in gastric cancer. ${ }^{175}$ Circ0010882 regulates biological functions by promoting $\mathrm{PI} 3 \mathrm{~K} / \mathrm{AKT} / \mathrm{mTOR}$ signaling. ${ }^{176}$ Further studies have 


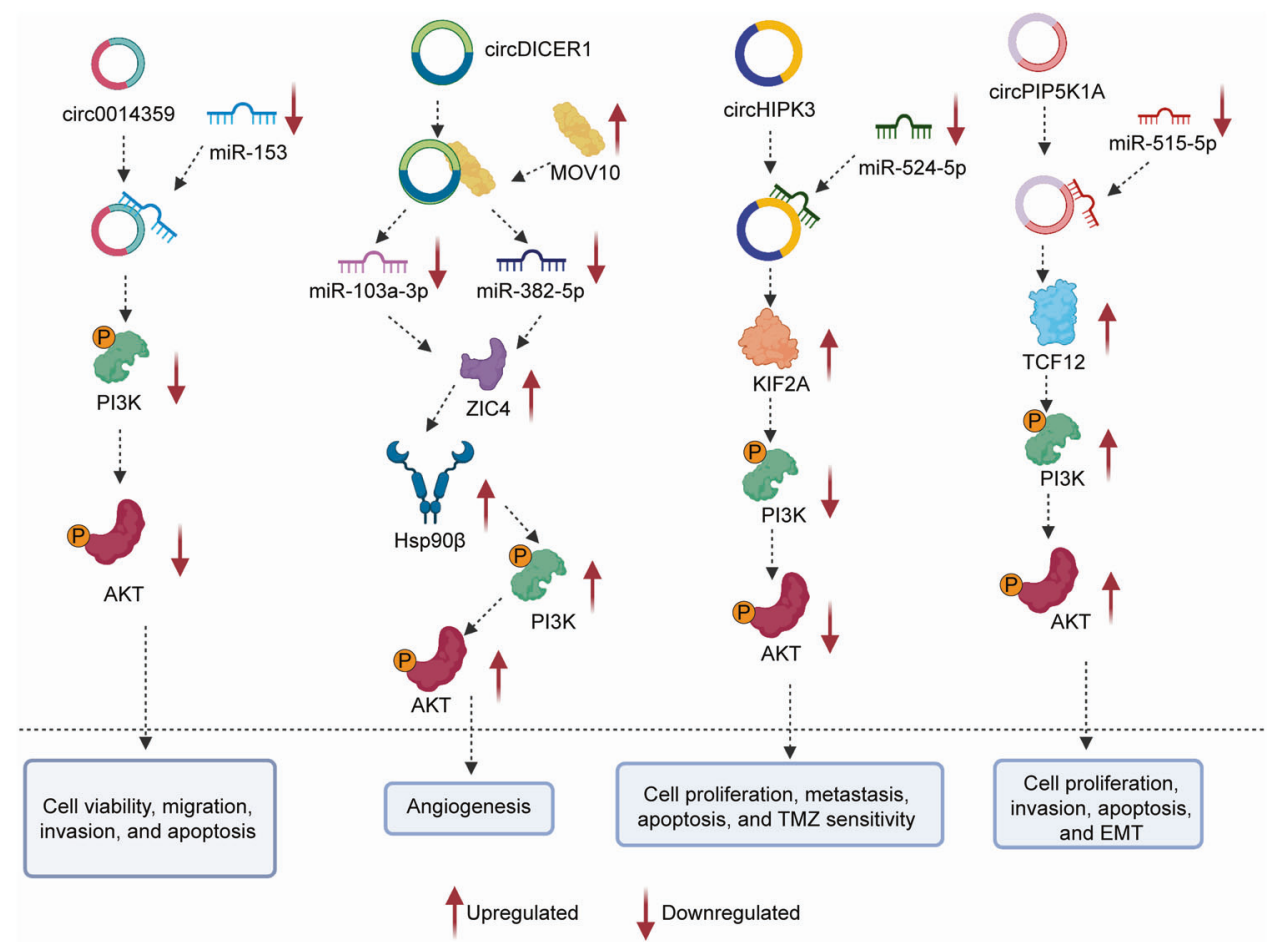

Fig. 3 The specific mechanism of glioma progression between circRNAs and PI3K/AKT pathway. Circ0014359 exerts its effects by inhibiting the level of miR-153 and regulating the PI3K/AKT axis. CircDICER1 in combination with MOV10 plays a critical role in glioma angiogenesis via regulation of miR-103a-3p (miR-382-5p)/ZIC4. CircHIPK3 regulates biological functions to improve sensitivity to temozolomide through suppression of the miR-524-5p/KIF2A-mediated PI3K/AKT pathway. circRNAs can also facilitate glioma tumorigenesis and progression by regulating the circPIP5K1A/miR-515-5p/TCF12/PI3K/AKT axis in glioma. Image created with BioRender (https://biorender.com/)

demonstrated that circ0023409, ciRS-7, circMAN2B2, and circPVT1 regulate the PI3K/AKT pathway by acting as sponges of miRNAs in gastric cancer. ${ }^{177-180}$ For example, circ0023409 activates the PI3K/ AKT pathway by sponging miR-542-3p to increase IRS4 levels. ${ }^{177}$ In addition, researchers have established in vivo xenograft nude mouse models to further explore the relationship between gastric cancer and the circRNA/PI3K/AKT axis. The expression of circPIP5K1A facilitates tumor growth in gastric cancer in vivo. ${ }^{175}$

\section{Colorectal cancer (CRC)}

The expression level of circ0001313 is dramatically upregulated while the levels of circCDYL and circ0008285 are decreased in CRC. $^{181-183}$ Circ0008285 expression is positively associated with LNM, tumor-node-metastasis (TNM) stage, and tumor size in patients with CRC. ${ }^{183}$ Functionally, circCDYL inhibits CRC cell migration and invasion. ${ }^{182}$ Circ0001313 and circCDYL significantly reduce cell apoptosis in CRC. ${ }^{181,182}$ Silencing the expression of circ0008285 enhances cell proliferation and migration in CRC. ${ }^{183}$ The expression of circ0001313 increases the level of AKT2, thus contributing to CRC progression by downregulating miR-510-5p expression. $^{181}$ CirCCDYL inactivates PI3K/AKT and JAK/STAT signaling by decreasing miR-150-5p levels in colon cancer. ${ }^{182}$ Circ0008285 expression reduces migration and proliferation via regulation of the miR-382-5p/PTEN/PI3K/AKT axis in CRC. ${ }^{183}$

\section{Liver cancer}

A series of circRNAs related to the circRNA/PI3K/AKT axis has been found to be closely related to the occurrence and progression of hepatocellular carcinoma (HCC). These circRNAs with aberrant expression are listed in Table $1 .^{184-190}$ Tumor size positively correlates with the expression of circlGF1R, circ0004001, circ0004123, and circ0075792 in HCC. ${ }^{185,188}$ High expression of circ0072309 is related to better 5-year survival in patients with HCC. ${ }^{186}$ Decreased circCDK13 levels enhance cell motility while low levels of circlGF1R inhibit cell growth in HCC. ${ }^{184,185}$ High expression of circ0072309 impairs cell growth and motility, affecting cell viability, colony formation, invasion, and migration. ${ }^{186}$ Mechanistically, circCDK13 inhibits HCC progression by regulating the PI3K/AKT and JAK/STAT pathways (Table 1). ${ }^{184}$ Circ0072309 functions as a sponge of miR-665 to negatively regulate the $\mathrm{PI} 3 \mathrm{~K} / \mathrm{AKT}$ and $\mathrm{Wnt} / \beta$-catenin pathways in the pathophysiologic processes of HCC. ${ }^{186}$ The expression of cirCEPHB4 impedes HCC progression by negatively regulating the HIF-1a/PI3K/AKT axis and HIF-1a/ZEB1 pathway. ${ }^{189}$ Hepatoblastoma is the most common primary malignant hepatic tumor in children. ${ }^{191,192}$ The expression of circHMGCS1 is significantly upregulated in hepatoblastoma cell lines compared to normal hepatocyte cells and HCC cells. ${ }^{193}$ circHMGCS1 also promotes cell proliferation and inhibits apoptosis in hepatoblastoma cell lines. CircHMGCS1 markedly upregulates the IGF2/IGF1R/PI3K/AKT axis to regulate proliferation by sponging miR-503-5p. ${ }^{193}$ The expression of circEPHB4 was negatively associated with tumor weight, size, and metastatic foci in vivo. ${ }^{89}$ A higher level of circ0079929 predicted decreased tumor size and weight in nude mouse models. ${ }^{187}$ CircCDK13 is an important negative regulator in the development and progression of HCC. ${ }^{184}$

Pancreatic cancer

The level of circNFIB1 is markedly decreased while circEIF6 and circBFAR expression levels are elevated in pancreatic cancer. ${ }^{194-196}$ High expression of circNFIB1 restrains lymphatic metastasis of pancreatic cancer. ${ }^{194}$ Upregulated levels of circBFAR predict high TNM stage and poor prognosis. ${ }^{196}$ Functionally, we found that the expression of circEIF6 promotes cell proliferation, increases cell migration and invasion, and inhibits cell apoptosis by performing siRNA-mediated knockdown experiments in pancreatic cancer cells. ${ }^{195}$ Mechanistically, circNFIB1 induces VEGF-C inhibition and attenuates LNM by sponging miR-486-5p and inhibiting the PI3K/ 
AKT pathway in pancreatic ductal adenocarcinoma. ${ }^{194}$ CircEIF6 regulates biological functions by upregulating miR-557 expression, downregulating SLC7A11 levels, and inactivating the PI3K/AKT pathway in pancreatic cancer. ${ }^{195}$ CircBFAR facilitates mesenchymal-epithelial transition by sponging miR-34b-5p and upregulating the MET/PI3K/AKT axis in pancreatic ductal adenocarcinoma. ${ }^{196}$ In vivo experiments showed that downregulation of circBFAR or circEIF6 expression can lead to lower tumor weight and volume in pancreatic ductal adenocarcinoma. ${ }^{195}$

\section{NERVOUS SYSTEM NEOPLASMS}

Glioma

PI3K/AKT axis-associated circRNAs are significantly upregulated in glioma (Table 1). ${ }^{197-202}$ Elevated circPIP5K1A expression is positively correlated with shorter survival time, larger tumor volume, and higher tumor stage in patients with glioma. ${ }^{200}$ CircHIPK3, circPIP5K1A, circ104075, and circ0000215 increase glioma cell proliferation in vitro. ${ }^{197,199,200,202}$ Cic0014359, circHIPK3, circPIP5K1A, and circ0000215 facilitate cell motility in glioma. ${ }^{197,199,200,202}$ Furthermore, circDICER1 markedly attenuates the angiogenesis of glioma-exposed endothelial cells. ${ }^{198}$ Downregulated expression of circHIPK3 induces a significant upregulation of temozolomide sensitivity in glioma. ${ }^{199}$ Mechanistic studies have revealed that circ0014359 exerts its effects by inhibiting the level of miR-153 and regulating the PI3K axis in glioma ${ }^{197}$ (Fig. 3). CircDICER1 in combination with MOV10 plays a critical role in glioma angiogenesis via regulation of miR-103a-3p (miR-382-5p)/ ZIC4. ${ }^{198}$ CircHIPK3 regulates biological functions to improve sensitivity to temozolomide through suppression of the miR524-5p/KIF2A-mediated PI3K/AKT pathway. ${ }^{199}$

A series of studies have shown that circRNAs can facilitate glioma tumorigenesis and progression by regulating the circPIP5K1A/miR515-5p/TCF12/PI3K/AKT and circ0000215/miR-495-3p/CXCR2/PI3K/
AKT pathways 200,202 (Fig. 3). Glioblastoma (GBM) is the most malignant glioma and has an extremely poor prognosis. ${ }^{203-205}$ CircAKT3 is overexpressed while circ0067934 and circPVT1 expression are significantly downregulated in GBM. ${ }^{62,206,207}$ A higher level of circ0067934 portends shorter disease-free survival and decreased overall survival rates in $\mathrm{GBM}^{206}$ Inhibition of circ0067934 expression may be a promising strategy for improving GBM prognosis. The upregulation of circAKT3 suppresses GBM cell proliferation and increases sensitivity to radiation. ${ }^{62}$ The expression of circ0067934 facilitates cell proliferation and metastasis and inhibits cell apoptosis in GBM by upregulating the PI3K-AKT pathway. ${ }^{206}$

Neuroblastoma (NB) and pituitary tumor

NB is the most common extracranial solid tumor in childhood. ${ }^{208-210}$ The expression of circ0002343 was found to be involved in the regulation of EMT in NB. ${ }^{211}$ circ0002343 significantly affects EMT by regulating the RAC1/PI3K/AKT/mTOR axis. Pituitary tumors are some of the most common benign neoplasms of the central nervous system. ${ }^{212,213}$ The levels of circ0054722, circ0012346, and circ0007362 are significantly increased while the expression of some circRNAs (circ0062222, circ0016403, circ0033349, and circ0049730) is downregulated in invasive nonfunctioning pituitary adenomas compared with the levels in noninvasive nonfunctioning pituitary adenomas. $^{214}$

\section{Genitourinary tumors}

Kidney cancer and bladder cancer. Kidney cancer is not a single disease but comprises different types of cancer that occur in the kidney. ${ }^{215-217}$ Renal carcinoma-associated transcripts (circ0072309 and circC3P1) are significantly downregulated in renal carcinoma tissues compared to corresponding normal tissues. ${ }^{218,219}$ These circRNAs significantly suppresses cell proliferation, migration, and invasion and promote cell apoptosis in kidney cancer. Circ0072309 sponges miR-100 to inhibit the PI3K/AKT and mTOR
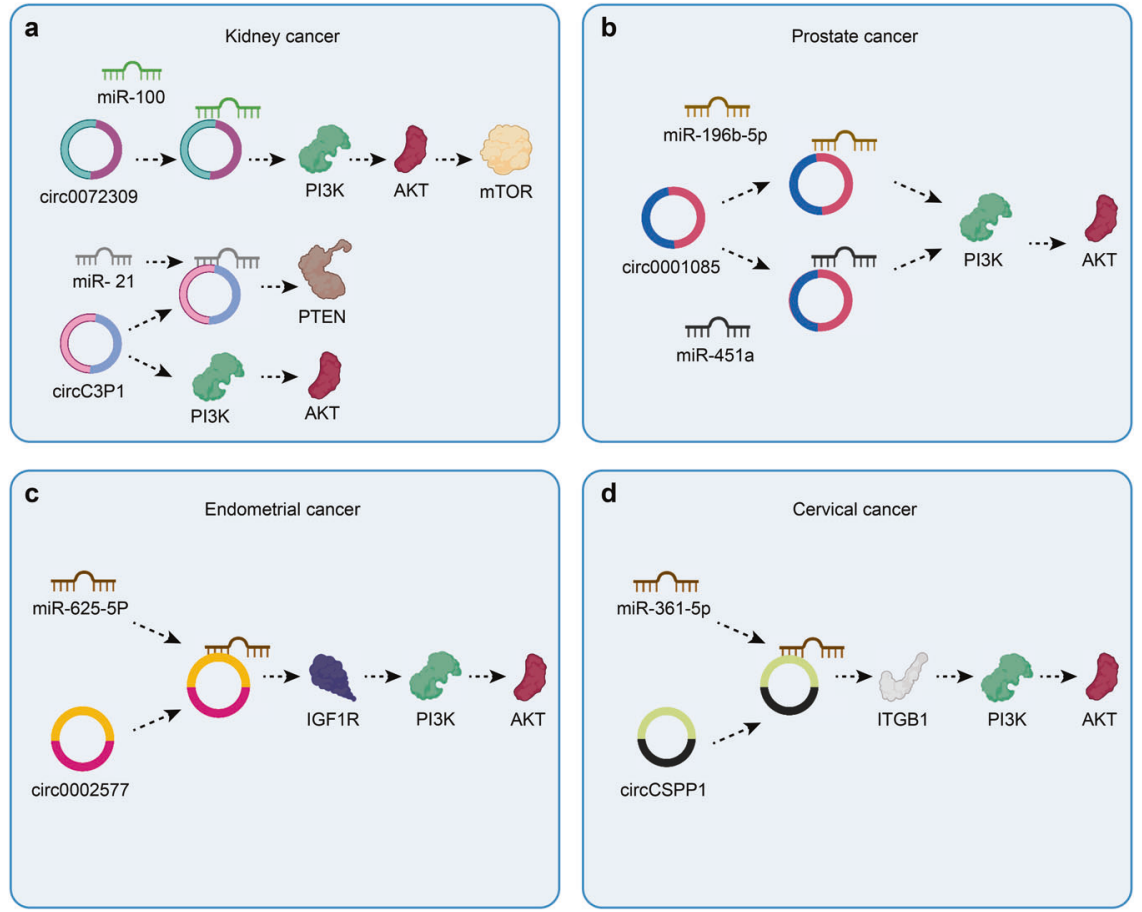

Fig. 4 The specific mechanism of circRNAs and PI3K/AKT pathway in different cancers. a Circ-0072309 sponges miR-100 to inhibit the PI3K/ AKT/mTOR pathway in kidney cancer. CircC3P1 inhibits kidney cancer progression via regulation of miR/PTEN pathways and the PI3K/AKT pathway. b Circ0001085 regulates prostate cancer progression through the PI3K/AKT pathway by sponging miR-196b-5p and miR-451a. c Overexpression of circ0002577 enhances the IGF1R/PI3K/AKT axis to increase the migration, invasion, and proliferation of endometrial cancer cells. d CircCSPP1 expression inhibits cervical cancer cell apoptosis and promotes cell proliferation and migration via the miR-361-5p/ ITGB1/PI3K/AKT axis in cervical cancer. Image created with BioRender (https://biorender.com/) 
10

pathways in kidney cancer. ${ }^{218}$ CircC3P1 exerts diverse biological functions by inhibiting the PI3K/AKT and NF-KB pathways by regulating the miR-21/PTEN axis ${ }^{219}$ (Fig. 4a). The overexpression of circZNF139 is markedly associated with disease-free survival in bladder cancer. ${ }^{220}$ circZNF139 overexpression also attenuates bladder cancer cell proliferation, colony formation, migration, and invasion by regulating the PI3K/AKT pathway.

Prostate cancer $(P C a)$. PCa is a major cause of male cancerrelated mortality worldwide. ${ }^{221-224}$ The level of circNOLC1 is increased while circlTCH expression is obviously downregulated in $\mathrm{PCa} .{ }^{225,226}$ CircMBOAT2 is overexpressed in PCa and contributes to poor prognosis. ${ }^{227}$ Moreover, increased circMBOAT2 levels are positively correlated with Gleason score and pathological T stage. Functionally, circNOLC1, circlTCH, and circMBOAT2 govern multiple cellular processes, such as cell proliferation, migration, and invasion, via the circRNA/PI3K/AKT axis in PCa. ${ }^{225-227}$ Circ0001085 induces EMT in PCa cells in vitro. ${ }^{228}$ Circ0001085 regulates PCa progression through the PI3K/AKT pathway by sponging miR196b-5p and miR-451a (Fig. 4b). CircMBOAT2 clearly promotes tumorigenesis and metastasis in PCa in vivo. ${ }^{227}$

Female reproductive system cancers. Ovarian, endometrial, and cervical cancer are three major malignant tumors causing a severe threat to women's health. ${ }^{229,230}$ The downregulation of cirCRHOBTB3 not only attenuates cell proliferation and metastasis but also inhibits glycolysis by suppressing the PI3K/AKT pathway in ovarian cancer. ${ }^{231}$ Circ0002577 expression is markedly increased in endometrial cancer. ${ }^{232}$ Circ0002577 expression is positively correlated with the histological grade of the tumor, LNM, and lymph vascular space invasion. Studies have revealed that patients with high expression of circ0002577 have a poor prognosis. The overexpression of circ0002577 enhances the IGF1R/PI3K/AKT axis to increase the migration, invasion, and proliferation of endometrial cancer cells (Fig. 4c). Silencing of circ0002577 expression significantly inhibits the growth and metastasis of tumors in nude mouse models of endometrial cancer. ${ }^{232}$ The expression of circCSPP1 is markedly upregulated in cervical cancer tissues. ${ }^{233}$ CircCSPP1 expression inhibits cervical cancer cell apoptosis and promotes cell proliferation and migration via the miR-361-5p/ITGB1/PI3K/AKT axis in cervical cancer (Fig. 4d).

\section{TUMORS OF THE ENDOCRINE SYSTEM}

Thyroid cancer is the most common malignancy occurring in the endocrine system. ${ }^{234-237}$ The expression of circ0067934 and circpsd3 is upregulated whereas circ0007694 expression is downregulated in thyroid tumors. ${ }^{238-240}$ High circ0067934 expression is associated with a shorter survival period of thyroid cancer patients. ${ }^{238}$ The expression of circ0067934 and circ0007694 affects diverse cell biological functions, such as cell proliferation, migration, invasion, and apoptosis, in thyroid cancer via the PI3K/AKT signaling pathway. ${ }^{238,239}$ During the regulation of different cellular biological processes, circ0067934 acts as an oncogene, but circ0007694 may function as a tumor suppressor gene in the progression of thyroid cancer. Increased circ0007694 expression effectively suppresses the growth of papillary thyroid carcinoma in vivo. ${ }^{239}$

\section{TUMORS OF THE RESPIRATORY AND MUSCULOSKELETAL SYSTEMS}

\section{Lung cancer}

Lung cancer is one of the leading causes of cancer-related death worldwide, with NSCLC accounting for $85 \%$ of all lung cancers. ${ }^{241-244}$ The expression of circGFRA1 and circ0018818 is significantly upregulated in NSCLC tissues compared to normal counterparts. $^{245,246}$ Silencing of circ0018818 expression inhibits proliferation, invasion, and EMT and promotes cell apoptosis. ${ }^{246}$ In addition, circGFRA1 activates the PI3K/AKT pathway by downregulating the expression of miR-188-3p in lung cancer. Knockdown of circ100876 reduces cell proliferation, migration, and invasion and facilitates NSCLC cell apoptosis by regulating the miR-636/RET axis and PI3K/ AKT signaling. ${ }^{247}$ The circ0018818/miR-767-3p/NID1/PI3K/AKT axis also plays a key role in the progression of lung cancer (Fig. 5).

Osteosarcoma (OS). OS is the most common primary malignant bone tumor in children and adolescents. ${ }^{248,249}$ The expression of circRNAs associated with the PI3K/AKT axis is listed in Table $1 .{ }^{250-253}$

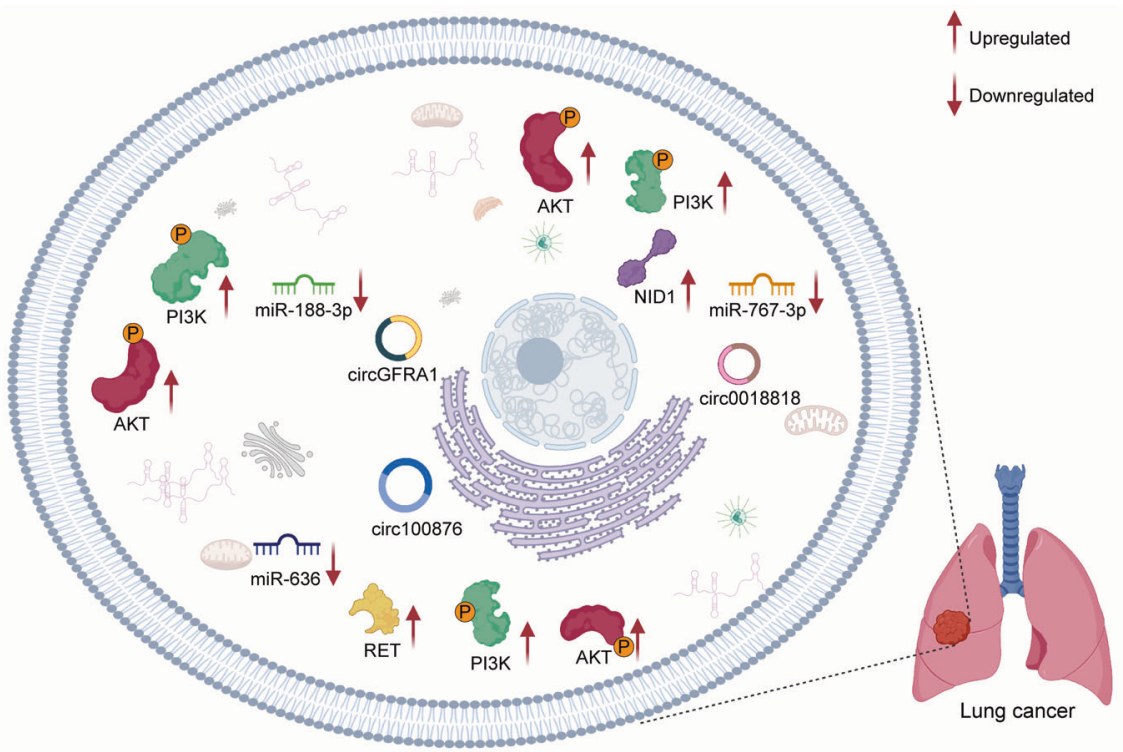

Fig. 5 The mechanism of circRNAs and PI3K/AKT pathway in lung cancer. CircGFRA1 activates the PI3K/AKT pathway by downregulating the expression of miR-188-3p in lung cancer. Circ100876 affects biological functions via PI3K/AKT signaling by regulating the miR-636/RET axis. The circ0018818/miR-767-3p/NID1/PI3K/AKT axis also plays a key role in the progression of lung cancer. Image created with BioRender (https:// biorender.com/) 
The expression of circEIF4G2 and circlTCH affects cell biological functions, such as cell proliferation, migration, and invasion, in OS. ${ }^{251,252}$ Silencing of circ0005909 obviously decreases cell viability and cell clone capacity in OS cell lines. ${ }^{253}$ Decreased expression of circ0001785 reduces cell proliferation and facilitates cell apoptosis in OS. ${ }^{250}$ Mechanistically, the expression of circ-ITCH attenuates cell biological functions because circ-ITCH acts as a competing endogenous RNA (ceRNA) for miR-22 to inactivate the PTEN/PI3K/ AKT and SP-1 pathways in OS. ${ }^{252}$ Circ0005909 expression enhances OS malignant progression by upregulating the MAPK-ERK and PI3KAkt signaling pathways by sponging miR-338-3p to inhibit the level of HGMA1. ${ }^{253}$

Tumors of other systems

Oral squamous cell carcinoma (OSCC) is a malignant type of head and neck squamous cell carcinoma. ${ }^{254-256}$ Circ043621 expression is remarkably elevated and circ102459 levels are dramatically decreased in OSCC tissues. ${ }^{256}$ CircPARD3 and circ043621 expression levels are relatively associated with clinical stage, LNM, and differentiation degree in OSCC. In vitro assays have revealed that increased circ043621 levels and decreased circ102459 expression can induce arrest in the G0 and/or G1 phase, apoptosis, and inhibition of cell proliferation by activating the MAPK and PI3K/AKT pathways. ${ }^{257}$ Multiple myeloma (MM) is a plasma cell malignancy. ${ }^{258-260}$ The expression of circ0007841 is significantly upregulated in MM cell lines and bone marrow-derived cells. ${ }^{261}$ High circ0007841 expression enhances the malignant behaviors of MM cells, for example, promoting cell proliferation, cell cycle progression, and metastasis, by activating the PI3K/AKT pathway.

$\mathrm{PIBK} / \mathrm{AKT}$ axis-associated circRNAs are aberrantly regulated in breast cancer ${ }^{262-267}$ (Table 1). The overexpression of circ0000199 is significantly associated with tumor size, TNM stage, and Ki-67 level in patients with breast cancer. ${ }^{267}$ Higher levels of circPRMT5, circHIPK3, circ001569, and circ0000199 predict poor prognosis in breast cancer. ${ }^{263,264,266,267}$ circ0000199 can affect tumor cell tolerance of chemotherapy via suppression of the PI3K/AKT/mTOR pathway and activation of the miR-206/miR-613 axis. ${ }^{267}$ circ0000199 also enhances cell proliferation, migration, and invasion in breast cancer. Silencing of circPRMT5 expression attenuates angiogenesis and proliferation and induces apoptosis. ${ }^{263}$ CircPRMT5 contributes to malignant phenotypes by activating the PI3K/AKT/mTOR axis via regulation of the miR-509-3p/TCF7L2 pathway. High expression of cirCHIPK3 significantly promotes cell migration, invasion, viability, and proliferation by targeting the miR-193a/HMGB1/PI3K/AKT axis. ${ }^{264}$ High circ0000442 expression induces suppression of cell viability and cell cycle arrest at the $\mathrm{G} 1$ phase and decreases colony formation in breast cancer. ${ }^{265}$ circ0000442 knockdown experiments have further confirmed this result. circ0000442 acts as a sponge of miR-148b-3p to downregulate the PTEN/PI3K/AKT pathway to impede tumor progression. Moreover, the knockdown of cirCHIPK3 attenuates breast cancer growth in vivo. ${ }^{264}$

CircRNAs related to the PI3K/AKT pathway as biomarkers In recent years, researchers have focused on identifying effective molecular biomarkers to improve the early detection, monitoring, and prediction of therapy response in cancer patients. ${ }^{268-270}$ Technological advances have contributed to an up-to-date understanding of the roles of circRNAs in the initiation and progression of cancer. A growing number of circRNAs related to the PI3K/AKT pathway have been found to be potential biomarkers for the diagnosis, treatment, and prognostication of many cancers. In this section, we will further discuss the important role of circRNAs in clinical applications.

\section{Diagnostic biomarkers}

The diagnosis of cancer at an early stage is critical for effective treatment and monitoring. ${ }^{271,272} \mathrm{~A}$ critical factor of early diagnosis is the identification of diagnostic biomarkers. ${ }^{273-275}$ Many
circRNAs in the PI3K/AKT pathway have been identified as aberrantly expressed during the progression of different cancers (Table 1). For example, the expression of circCSPP1 is markedly upregulated in cervical cancer tissues. ${ }^{233}$ The expression of circGFRA1 and circ0018818 is significantly upregulated in NSCLC tissues compared to normal tissues. ${ }^{245,246}$ CircRNAs with significantly abnormal expression have diagnostic potential in many cancers. In addition, the levels of circ0004001, circ0004123, and circ0075792 in serum are markedly upregulated in patients with HCC. $^{188}$ The expression of circ0010882 in serum is obviously elevated in gastric cancer patients. ${ }^{176}$

The expression of circ0007841 in serum is significantly increased in MM patients. ${ }^{261}$ These results suggest that early diagnosis based on circRNAs is practical. More studies about the diagnostic roles of circRNAs in serum are needed.

\section{Prognosis prediction}

Emerging evidence suggests that many circRNAs are reliable for predicting the prognosis of patients with cancer. ${ }^{196,276,277}$ which provides important guidance for cancer therapy. A significant number of circRNAs have been found to be markedly associated with survival parameters, such as overall survival, disease-free survival, and the 5-year survival rate (Table 2). Low circVRK1 expression predicts poor overall survival in patients with ESCC. ${ }^{172}$ A higher level of circ0067934 portends shorter disease-free survival and decreased overall survival rates in GBM. ${ }^{206}$ The expression of circ0072309 is positively correlated with the 5-year survival rate in patients with liver cancer. ${ }^{186}$ In addition, some circRNAs have been found to be significantly associated with other clinical features in cancer. Elevated circLPAR3 levels are markedly associated with LNM and advanced TNM stage in esophageal cancer. ${ }^{171}$ The levels of circ0010882 and circ0023409 are positively associated with tumor size and histological grade in gastric cancer patients. ${ }^{176,177}$ The elevated expression of cirCMBOAT2 has positively correlated with the Gleason score and pathological T stage in $\mathrm{PCa}{ }^{227}$ These results provide an important reference for cancer treatment.

\section{Targeted therapies}

Targeted therapy, a recent trend in cancer therapy, is emerging as a novel therapeutic strategy. ${ }^{278-280}$ Targeted therapies significantly enhance the efficiency of cancer therapy. ${ }^{281,282}$ CircRNAs can positively or negatively modulate biological functions and cancer progression through multiple signaling pathways. CirCLARP4 promotes the expression of PTEN and inactivates the PI3K/ AKT pathway to suppress the progression of ESCC. ${ }^{173}$ CircPIP5K1A sponges miR-671-5p to facilitate tumor progression by upregulating KRT80 and the PI3K/AKT pathway in gastric cancer. ${ }^{175}$ Circ0067934 facilitates cell proliferation and metastasis and inhibits cell apoptosis in GBM by upregulating the PI3K-AKT pathway. ${ }^{206}$ CircEPHB4 impedes HCC progression by negatively regulating the HIF-1a/PI3K/AKT axis and the HIF-1a/ZEB1 pathway in HCC. ${ }^{189}$ Upregulating or downregulating the expression of circRNAs may be a feasible way to regulate tumor progression. Silencing of circ0010882 attenuates gastric cancer cell growth and motility in vitro. ${ }^{176}$ Knockdown of circ100876 reduces cell proliferation, migration, and invasion and facilitates NSCLC cell apoptosis. $^{247}$ In addition, a miR-671-5p inhibitor was able to significantly reduce the level of circPIP5K1A to inhibit the progression of gastric cancer. ${ }^{175}$ Rapamycin, an mTOR inhibitor, blocks the circMBOAT2/PI3K/AKT/mTOR pathway to suppress PCa progression. ${ }^{227}$ CircHIPK3 regulates biological functions to improve sensitivity to temozolomide through suppression of the miR-524-5p/KIF2A-mediated PI3K/AKT pathway in glioma. ${ }^{199}$ Circ0000199 can make tumor cells sensitive to chemotherapy via suppression of the $\mathrm{PI} 3 \mathrm{~K} / \mathrm{AKT} / \mathrm{mTOR}$ pathway and activation of the miR-206/miR-613 axis in breast cancer. ${ }^{267}$ High expression of circPVT1 enhances the sensitivity of gastric cancer cells to cisplatin 
(DDP). ${ }^{180}$ These results provide important information for the clinical treatment of cancers.

\section{CONCLUSIONS AND FUTURE PERSPECTIVES}

CircRNAs are emerging biomarkers in cancer diagnosis and treatment. Complex circRNA regulatory networks have important implications in cancer research and have revolutionized our views on cancer genesis, progression, and treatment. In terms of circRNA-mediated cellular signaling studies, the most exciting finding is that circRNAs can function through molecular associations with the components of classical signaling pathways. The $\mathrm{PI} 3 \mathrm{~K} / \mathrm{AKT}$ pathway is closely associated with the pathogenesis and development of cancer. It can regulate cell survival and proliferation and plays an essential role in cell migration, invasion, and angiogenesis. The circRNA/PI3K/AKT axis has recently attracted increasing attention. The modulating effect of tumor cellular biological functions is of interest for researchers studying the circRNA/PI3K/AKT axis. In terms of the circRNA/PI3K/AKT axis, plenty of circRNAs have been extensively studied. The ubiquitous expression and tumor specificity of circRNAs have ushered in new opportunities for cancer diagnosis. The expression of circRNAs is significantly associated with the clinical phenotype and survival time, indicating that it has important guiding significance for cancer prognostic evaluation. However, the expression level and expression stability of circRNAs in circulating body fluids need further study. Assessment of the expression stability of circRNAs in circulating body fluids, including urine and blood, has vast prospects in terms of clinical applications. In addition, considering the aberrant expression of a large number of cancer-related circRNAs, it is crucial to identify circRNAs related to certain types of cancer.

CircRNAs positively or negatively regulate biological functions in cancer development and progression via the PI3K/AKT signaling pathway. Thus, we may control the cancer process by regulating circRNAs in the circRNA/PI3K/AKT axis. The implementation of this idea relies on in-depth research of pharmacologic therapies. A promising drug must stably regulate circRNA activity and efficiently transduce the effect, thus controlling cancer progression. This necessitates a deeper understanding of the functions and mechanisms of circRNA related to the PI3K/AKT pathway under physiological and pathophysiological conditions. At present, research on the circRNA/PI3K/AKT axis is still in its infancy. Structural and functional data for circRNAs related to PI3K/AKT pathway remain limited. The mechanism of interactions between circRNAs and the PI3K/AKT pathway has yet to be established. Without detailed information on the structure and function of circRNAs, therapeutic options based on PI3K/AKT pathway are difficult to identify.

\section{ACKNOWLEDGEMENTS}

This work was funded by the National Natural Science Foundation of China (81790631), the Zhejiang University Academic Award for Outstanding Doctoral Candidates (2020055), the Independent Project Fund of the State Key Laboratory for Diagnosis and Treatment of Infectious Diseases, the National Key Research and Development Program of China (2016YFC1101404/3), and Zhejiang Basic Public Welfare Research Program of China (LQ20H030012).

\section{AUTHOR CONTRIBUTIONS}

Lanjuan Li designed the study and reviewed and edited the paper; Chen Xue and Ganglei Li participated in original draft preparation; Juan Lu collected the references and help with reviewing the paper; all authors have read and approved the article.

\section{ADDITIONAL INFORMATION}

Competing interests: The authors declare no competing interests.

\section{REFERENCES}

1. Andrei, L. et al. Advanced technological tools to study multidrug resistance in cancer. Drug Resist. Updat 48, 100658 (2020).

2. Gapstur, S. M. et al. A blueprint for the primary prevention of cancer: targeting established, modifiable risk factors. CA Cancer J. Clin. 68, 446-470 (2018).

3. Martínez-Jiménez, F. et al. A compendium of mutational cancer driver genes. Nat. Rev. Cancer 20, 555-572 (2020).

4. Alcaraz, K. I. et al. Understanding and addressing social determinants to advance cancer health equity in the United States: a blueprint for practice, research, and policy. CA Cancer J. Clin. 70, 31-46 (2020).

5. Yabroff, K. R., Gansler, T., Wender, R. C., Cullen, K. J. \& Brawley, O. W. Minimizing the burden of cancer in the United States: goals for a high-performing health care system. CA Cancer J. Clin. 69, 166-183 (2019).

6. Bright, C. J. et al. Risk of subsequent primary neoplasms in survivors of adolescent and young adult cancer (Teenage and Young Adult Cancer Survivor Study): a population-based, cohort study. Lancet Oncol. 20, 531-545 (2019).

7. Rupaimoole, R. \& Slack, F. J. MicroRNA therapeutics: towards a new era for the management of cancer and other diseases. Nat. Rev. Drug Discov. 16, 203-222 (2017).

8. Yin, H., Xue, W. \& Anderson, D. G. CRISPR-Cas: a tool for cancer research and therapeutics. Nat. Rev. Clin. Oncol. 16, 281-295 (2019).

9. Akhave, N. S., Biter, A. B. \& Hong, D. S. Mechanisms of resistance to KRAS(G12C)targeted therapy. Cancer Discov. 11, 1345-1352 (2021).

10. Evans, E. R., Bugga, P., Asthana, V. \& Drezek, R. Metallic nanoparticles for cancer immunotherapy. Mater. Today (Kidlington) 21, 673-685 (2018).

11. Aufiero, S., Reckman, Y. J., Pinto, Y. M. \& Creemers, E. E. Circular RNAs open a new chapter in cardiovascular biology. Nat. Rev. Cardiol. 16, 503-514 (2019).

12. Vo, J. N. et al. The landscape of circular RNA in cancer. Cell 176, 869-881.e813 (2019).

13. Zeng, Z. et al. Circular RNA CircMAP3K5 acts as a microRNA-22-3p sponge to promote resolution of intimal hyperplasia via TET2-mediated smooth muscle cell differentiation. Circulation 143, 354-371 (2021).

14. Wen, G., Zhou, T. \& Gu, W. The potential of using blood circular RNA as liquid biopsy biomarker for human diseases. Protein Cell 5, 1-36 (2020).

15. Chen, Y. G. et al. N6-methyladenosine modification controls circular RNA immunity. Mol. Cell 76, 96-109.e109 (2019).

16. Legnini, l. et al. Circ-ZNF609 is a circular RNA that can be translated and functions in myogenesis. Mol. Cell 66, 22-37.e29 (2017).

17. Zhang, Z. Y. et al. CircRNA_101237 promotes NSCLC progression via the miRNA490-3p/MAPK1 axis. Sci. Rep. 10, 9024 (2020).

18. Liu, Z. et al. Circular RNA clARS regulates ferroptosis in HCC cells through interacting with RNA binding protein ALKBH5. Cell Death Discov. 6, 72 (2020).

19. Bilanges, B., Posor, Y. \& Vanhaesebroeck, B. PI3K isoforms in cell signalling and vesicle trafficking. Nat. Rev. Mol. Cell Biol. 20, 515-534 (2019).

20. Molinaro, A. et al. Insulin-driven PI3K-AKT signaling in the hepatocyte is mediated by redundant PI3Ka and PI3K $\beta$ activities and is promoted by RAS. Cell Metab. 29, 1400-1409.e1405 (2019).

21. Hopkins, B. D., Goncalves, M. D. \& Cantley, L. C. Insulin-PI3K signalling: an evolutionarily insulated metabolic driver of cancer. Nat. Rev. Endocrinol. 16, 276-283 (2020).

22. Fruman, D. A. et al. The PI3K pathway in human disease. Cell 170, 605-635 (2017).

23. Hoxhaj, G. \& Manning, B. D. The PI3K-AKT network at the interface of oncogenic signalling and cancer metabolism. Nat. Rev. Cancer 20, 74-88 (2020).

24. Hanker, A. B., Kaklamani, V. \& Arteaga, C. L. Challenges for the clinical development of PI3K inhibitors: strategies to improve their impact in solid tumors. Cancer Discov. 9, 482-491 (2019).

25. Okkenhaug, K., Graupera, M. \& Vanhaesebroeck, B. Targeting PI3K in cancer: impact on tumor cells, their protective stroma, angiogenesis, and immunotherapy. Cancer Discov. 6, 1090-1105 (2016).

26. Le, X. et al. Systematic functional characterization of resistance to PI3K inhibition in breast cancer. Cancer Discov. 6, 1134-1147 (2016).

27. Fresno Vara, J. A. et al. PI3K/Akt signalling pathway and cancer. Cancer Treat. Rev. 30, 193-204 (2004).

28. Yuan, T. L. \& Cantley, L. C. Introduction. Curr. Top. Microbiol. Immunol. 346, 1-7 (2010).

29. Arafeh, R. \& Samuels, Y. PIK3CA in cancer: the past 30 years. Semin. Cancer Biol. 59, 36-49 (2019).

30. Gulluni, F., De Santis, M. C., Margaria, J. P., Martini, M. \& Hirsch, E. Class II PI3K functions in cell biology and disease. Trends Cell Biol. 29, 339-359 (2019).

31. Vanhaesebroeck, B., Guillermet-Guibert, J., Graupera, M. \& Bilanges, B. The emerging mechanisms of isoform-specific PI3K signalling. Nat. Rev. Mol. Cell Biol. 11, 329-341 (2010).

32. El Motiam, A. et al. SUMOylation modulates the stability and function of PI3Kp110ß. Cell Mol. Life Sci. 78, 4053-4065 (2021). 
33. de la Cruz-Herrera, C. F. et al. Conjugation of SUMO to p85 leads to a novel mechanism of PI3K regulation. Oncogene 35, 2873-2880 (2016).

34. Hofmann, B. T. \& Jücker, M. Activation of PI3K/Akt signaling by $n$-terminal $\mathrm{SH}_{2}$ domain mutants of the p85a regulatory subunit of PI3K is enhanced by deletion of its c-terminal SH2 domain. Cell Signal. 24, 1950-1954 (2012).

35. Shin, Y. K., Liu, Q., Tikoo, S. K., Babiuk, L. A. \& Zhou, Y. Influenza A virus NS1 protein activates the phosphatidylinositol 3-kinase (PI3K)/Akt pathway by direct interaction with the p85 subunit of PI3K. J. Gen. Virol. 88, 13-18 (2007).

36. Zhang, M., Jang, H. \& Nussinov, R. PI3K driver mutations: a biophysical membrane-centric perspective. Cancer Res. 81, 237-247 (2021).

37. Katan, M. \& Cockcroft, S. Phosphatidylinositol(4,5)bisphosphate: diverse functions at the plasma membrane. Essays Biochem. 64, 513-531 (2020).

38. Ishikawa, S. et al. Role of connexin-43 in protective PI3K-Akt-GSK-3 $\beta$ signaling in cardiomyocytes. Am. J. Physiol. Heart Circ. Physiol. 302, H2536-H2544 (2012).

39. Dey, J. H. et al. Targeting fibroblast growth factor receptors blocks PI3K/AKT signaling, induces apoptosis, and impairs mammary tumor outgrowth and metastasis. Cancer Res. 70, 4151-4162 (2010).

40. Chen, J., Nagle, A. M., Wang, Y. F., Boone, D. N. \& Lee, A. V. Controlled dimerization of insulin-like growth factor-1 and insulin receptors reveals shared and distinct activities of holo and hybrid receptors. J. Biol. Chem. 293, 3700-3709 (2018).

41. Murillo, M. M. et al. RAS interaction with PI3K p110a is required for tumorinduced angiogenesis. J. Clin. Investig. 124, 3601-3611 (2014).

42. Tamaskovic, R. et al. Intermolecular biparatopic trapping of ErbB2 prevents compensatory activation of PI3K/AKT via RAS-p110 crosstalk. Nat. Commun. 7, 11672 (2016).

43. Johnson, C., Chun-Jen Lin, C. \& Stern, M. Ras-dependent and Ras-independent effects of PI3K in Drosophila motor neurons. Genes Brain Behav. 11, 848-858 (2012).

44. Bresnick, A. R. \& Backer, J. M. PI3Kß-A versatile transducer for GPCR, RTK, and small GTPase signaling. Endocrinology 160, 536-555 (2019).

45. Oudit, G. Y. et al. The role of phosphoinositide-3 kinase and PTEN in cardiovascular physiology and disease. J. Mol. Cell Cardiol. 37, 449-471 (2004).

46. Houslay, D. M. et al. Coincident signals from GPCRs and receptor tyrosine kinases are uniquely transduced by PI3K $\beta$ in myeloid cells. Sci. Signal. 9, ra82 (2016).

47. Zhu, F. et al. PRMT5 is upregulated by B-cell receptor signaling and forms a positive-feedback loop with PI3K/AKT in lymphoma cells. Leukemia 33, 2898-2911 (2019)

48. Wang, L., Liu, W. X. \& Huang, X. G. MicroRNA-199a-3p inhibits angiogenesis by targeting the VEGF/PI3K/AKT signalling pathway in an in vitro model of diabetic retinopathy. Exp. Mol. Pathol. 116, 104488 (2020).

49. Duan, Y., Haybaeck, J. \& Yang, Z. Therapeutic potential of PI3K/AKT/mTOR pathway in gastrointestinal stromal tumors: rationale and progress. Cancers 12, 2972 (2020).

50. Damayanti, N. P. et al. Therapeutic targeting of TFE3/IRS- $1 / \mathrm{PI} 3 \mathrm{~K} / \mathrm{mTOR}$ axis in translocation renal cell carcinoma. Clin. Cancer Res. 24, 5977-5989 (2018).

51. Talbot, $\mathrm{K}$. et al. Demonstrated brain insulin resistance in Alzheimer's disease patients is associated with IGF-1 resistance, IRS-1 dysregulation, and cognitive decline. J. Clin. Investig. 122, 1316-1338 (2012).

52. Guo, H. et al. The PI3K/AKT pathway and renal cell carcinoma. J. Genet. Genomics 42, 343-353 (2015).

53. Yang, Q., Jiang, W. \& Hou, P. Emerging role of PI3K/AKT in tumor-related epigenetic regulation. Semin. Cancer Biol. 59, 112-124 (2019).

54. Lien, E. C., Dibble, C. C. \& Toker, A. PI3K signaling in cancer: beyond AKT. Curr. Opin. Cell Biol. 45, 62-71 (2017).

55. Manning, B. D. \& Toker, A. AKT/PKB signaling: navigating the network. Cell 169 381-405 (2017).

56. Manning, B. D. \& Cantley, L. C. AKT/PKB signaling: navigating downstream. Cell 129, 1261-1274 (2007).

57. Pearce, L. R., Komander, D. \& Alessi, D. R. The nuts and bolts of AGC protein kinases. Nat. Rev. Mol. Cell Biol. 11, 9-22 (2010).

58. Revathidevi, S. \& Munirajan, A. K. Akt in cancer: mediator and more. Semin. Cancer Biol. 59, 80-91 (2019).

59. Leroux, A. E., Schulze, J. O. \& Biondi, R. M. AGC kinases, mechanisms of regulation and innovative drug development. Semin. Cancer Biol. 48, 1-17 (2018).

60. Palumbo, S., Paterson, C., Yang, F., Hood, V. L. \& Law, A. J. PKB $\beta / A K T 2$ deficiency impacts brain mTOR signaling, prefrontal cortical physiology, hippocampal plasticity and select murine behaviors. Mol. Psychiatry 26, 411-428 (2021).

61. Hinz, N. \& Jücker, M. Distinct functions of AKT isoforms in breast cancer: a comprehensive review. Cell Commun. Signal. 17, 154 (2019).

62. Xia, X. et al. A novel tumor suppressor protein encoded by circular AKT3 RNA inhibits glioblastoma tumorigenicity by competing with active phosphoinositide-dependent Kinase-1. Mol. Cancer 18, 131 (2019).
63. Häggblad Sahlberg, S. et al. Different functions of AKT1 and AKT2 in molecular pathways, cell migration and metabolism in colon cancer cells. Int J. Oncol. 50, 5-14 (2017).

64. Yang, S. et al. The flavonoid baicalin improves glucose metabolism by targeting the PH domain of AKT and activating AKT/GSK3 $\beta$ phosphorylation. FEBS Lett. 593, 175-186 (2019).

65. Takenaka, N., Nakao, M., Matsui, S. \& Satoh, T. A crucial role for the small GTPase Rac1 downstream of the protein kinase Akt2 in insulin signaling that regulates glucose uptake in mouse adipocytes. Int. J. Mol. Sci. 20, 5443 (2019).

66. Zhang, D., Wang, J., Zhou, C. \& Xiao, W. Zebrafish akt2 is essential for survival, growth, bone development, and glucose homeostasis. Mech. Dev. 143, 42-52 (2017).

67. Wen, L. et al. Characterization of AKT somatic mutations in Chinese breast cancer patients. Cancer Manag. Res. 13, 3055-3065 (2021).

68. Kumar, S. et al. Spectrum of PIK3CA/AKT mutations across molecular subtypes of triple-negative breast cancer. Breast Cancer Res. Treat. 187, 625-633 (2021).

69. Conduit, S. E. et al. AKT signaling promotes DNA damage accumulation and proliferation in polycystic kidney disease. Hum. Mol. Genet. 29, 31-48 (2020).

70. Lei, N., Peng, B. \& Zhang, J. Y. CIP2A regulates cell proliferation via the AKT signaling pathway in human lung cancer. Oncol. Rep. 32, 1689-1694 (2014).

71. Lee, J. H. et al. Stabilization of phosphofructokinase 1 platelet isoform by AKT promotes tumorigenesis. Nat. Commun. 8, 949 (2017).

72. Dimmeler, S. \& Zeiher, A. M. Akt takes center stage in angiogenesis signaling Circ. Res. 86, 4-5 (2000).

73. Montaner, S. Akt/TSC/mTOR activation by the KSHV G protein-coupled receptor: emerging insights into the molecular oncogenesis and treatment of Kaposi's sarcoma. Cell Cycle 6, 438-443 (2007).

74. Huang, $H$. et al. Hepatitis $C$ virus inhibits AKT-tuberous sclerosis complex (TSC), the mechanistic target of rapamycin (mTOR) pathway, through endoplasmic reticulum stress to induce autophagy. Autophagy 9, 175-195 (2013).

75. Kumar, P., Raman, T., Swain, M. M., Mishra, R. \& Pal, A. Hyperglycemia-induced oxidative-nitrosative stress induces inflammation and neurodegeneration via augmented tuberous sclerosis complex-2 (TSC-2) activation in neuronal cells. Mol. Neurobiol. 54, 238-254 (2017).

76. Li, T. et al. P21 and P27 promote tumorigenesis and progression via cell cycle acceleration in seminal vesicles of TRAMP mice. Int. J. Biol. Sci. 15, 2198-2210 (2019).

77. Yip, W. K., Leong, V. C., Abdullah, M. A., Yusoff, S. \& Seow, H. F. Overexpression of phospho-Akt correlates with phosphorylation of EGF receptor, FKHR and BAD in nasopharyngeal carcinoma. Oncol. Rep. 19, 319-328 (2008).

78. Barati, M. T., Scherzer, J., Wu, R., Rane, M. J. \& Klein, J. B. Cytoskeletal rearrangement and Src and PI-3K-dependent Akt activation control GABA(B)R-mediated chemotaxis. Cell Signal. 27, 1178-1185 (2015).

79. Calandria, J. M. et al. Ataxin-1 poly(Q)-induced proteotoxic stress and apoptosis are attenuated in neural cells by docosahexaenoic acid-derived neuroprotectin D1. J. Biol. Chem. 287, 23726-23739 (2012).

80. $\mathrm{Wu}, \mathrm{S}$. et al. Human adipose-derived mesenchymal stem cells promote breast cancer MCF7 cell epithelial-mesenchymal transition by cross interacting with the TGF- $\beta /$ Smad and PI3K/AKT signaling pathways. Mol. Med. Rep. 19, 177-186 (2019).

81. Jeong, S. H., Yang, M. J., Choi, S., Kim, J. \& Koh, G. Y. Refractoriness of STING therapy is relieved by AKT inhibitor through effective vascular disruption in tumour. Nat. Commun. 12, 4405 (2021).

82. Wang, K., Liang, Y., Su, Y. \& Wang, L. DhHP-6 ameliorates hepatic oxidative stress and insulin resistance in type 2 diabetes mellitus through the PI3K/AKT and AMPK pathway. Biochem. J. 477, 2363-2381 (2020).

83. Lv, H., Li, J. \& Che, Y. Q. CXCL8 gene silencing promotes neuroglial cells activation while inhibiting neuroinflammation through the PI3K/Akt/NF-KB-signaling pathway in mice with ischemic stroke. J. Cell Physiol. 234, 7341-7355 (2019).

84. Ersahin, T., Tuncbag, N. \& Cetin-Atalay, R. The PI3K/AKT/mTOR interactive pathway. Mol. Biosyst. 11, 1946-1954 (2015).

85. Karar, J. \& Maity, A. PI3K/AKT/mTOR pathway in angiogenesis. Front. Mol. Neurosci. 4, 51 (2011)

86. Tessier, M. \& Woodgett, J. R. Serum and glucocorticoid-regulated protein kinases: variations on a theme. J. Cell Biochem. 98, 1391-1407 (2006).

87. Yang, Y. et al. Leep1 interacts with PIP3 and the Scar/WAVE complex to regulate cell migration and macropinocytosis. J. Cell Biol. 220, e202010096 (2021).

88. Li, H. \& Marshall, A. J. Phosphatidylinositol $(3,4)$ bisphosphate-specific phosphatases and effector proteins: a distinct branch of PI3K signaling. Cell Signal. 27, 1789-1798 (2015).

89. Dieterle, A. M. et al. PDK1 controls upstream PI3K expression and PIP3 generation. Oncogene 33, 3043-3053 (2014).

90. Misra, U. K. \& Pizzo, S. V. Activated a2-macroglobulin binding to cell surface GRP78 induces T-loop phosphorylation of Akt1 by PDK1 in association with Raptor. PLOS ONE 9, e88373 (2014). 
91. Dangelmaier, C. et al. PDK1 selectively phosphorylates $\operatorname{Thr}(308)$ on Akt and contributes to human platelet functional responses. Thromb. Haemost. 111, 508-517 (2014).

92. Kawakami, Y. et al. Protein kinase $C$ betall regulates Akt phosphorylation on Ser473 in a cell type- and stimulus-specific fashion. J. Biol. Chem. 279, 47720-47725 (2004).

93. Hresko, R. C., Murata, H. \& Mueckler, M. Phosphoinositide-dependent kinase-2 is a distinct protein kinase enriched in a novel cytoskeletal fraction associated with adipocyte plasma membranes. J. Biol. Chem. 278, 21615-21622 (2003).

94. Zhao, J. et al. 7,8-Dihydroxyflavone suppresses proliferation and induces apoptosis of human osteosarcoma cells. Acta Biochim. Biophys. Sin. 53, 903-911 (2021).

95. Zhu, S. et al. 20(S)-ginsenoside $\mathrm{Rh} 2$ induces caspase-dependent promyelocytic leukemia-retinoic acid receptor A degradation in NB4 cells via Akt/Bax/caspase9 and TNF-a/caspase8 signaling cascades. J. Ginseng Res. 45, 295-304 (2021).

96. Zhang, B. et al. MiR-217 inhibits apoptosis of atherosclerotic endothelial cells via the TLR4/PI3K/Akt/NF-KB pathway. Eur. Rev. Med. Pharm. Sci. 24, 12867-12877 (2020).

97. Liu, Y. C. et al. Lenvatinib inhibits AKT/NF-KB signaling and induces apoptosis through extrinsic/intrinsic pathways in non-small cell lung cancer. Anticancer Res. 41, 123-130 (2021).

98. Duda, P. et al. GSK-3 and miRs: master regulators of therapeutic sensitivity of cancer cells. Biochim. Biophys. Acta Mol. Cell Res. 1867, 118770 (2020).

99. Shang, J., Gao, Z. Y., Zhang, L. Y. \& Wang, C. Y. Over-expression of JAZF1 promotes cardiac microvascular endothelial cell proliferation and angiogenesis via activation of the Akt signaling pathway in rats with myocardial ischemiareperfusion. Cell Cycle 18, 1619-1634 (2019).

100. Wang, L. et al. MiR-4787-5p regulates vascular smooth muscle cell apoptosis by targeting PKD1 and inhibiting the PI3K/Akt/FKHR pathway. J. Cardiovasc. Pharmacol. 78, 288-296 (2021).

101. Mu, M., Niu, W., Zhang, X., Hu, S. \& Niu, C. LncRNA BCYRN1 inhibits glioma tumorigenesis by competitively binding with miR-619-5p to regulate CUEDC2 expression and the PTEN/AKT/p21 pathway. Oncogene 39, 6879-6892 (2020).

102. Chibaya, L., Karim, B., Zhang, H. \& Jones, S. N. Mdm2 phosphorylation by Akt regulates the $\mathrm{p} 53$ response to oxidative stress to promote cell proliferation and tumorigenesis. Proc Natl Acad Sci USA 118, e2003193118 (2021).

103. Li, K. et al. High cholesterol induces apoptosis and autophagy through the ROSactivated AKT/FOXO1 pathway in tendon-derived stem cells. Stem Cell Res. Ther. 11, 131 (2020).

104. Huang, Y. et al. Effect of miR-182 on hepatic fibrosis induced by Schistosomiasis japonica by targeting FOXO1 through PI3K/AKT signaling pathway. J. Cell Physiol. 233, 6693-6704 (2018).

105. Yu, L., Wei, J. \& Liu, P. Attacking the PI3K/Akt/mTOR signaling pathway for targeted therapeutic treatment in human cancer. Semin. Cancer Biol. S1044579X, 00188-7 (2021).

106. Chen, J., Alduais, Y., Zhang, K., Zhu, X. \& Chen, B. CCAT1/FABP5 promotes tumour progression through mediating fatty acid metabolism and stabilizing $\mathrm{PI} 3 \mathrm{~K} / \mathrm{AKT} / \mathrm{mTOR}$ signalling in lung adenocarcinoma. J. Cell. Mol. Med. 25, 9199-9213 (2021).

107. Du, W. et al. Methylation of NRN1 is a novel synthetic lethal marker of PI3K-AktmTOR and ATR inhibitors in esophageal cancer. Cancer Sci. 112, 2870-2883 (2021).

108. $\mathrm{XU}$, L. et al. SLC1A3 promotes gastric cancer progression via the PI3K/AKT signalling pathway. J. Cell Mol. Med. 24, 14392-14404 (2020).

109. Qu, J. et al. AKR1B10 promotes breast cancer cell proliferation and migration via the PI3K/AKT/NF-KB signaling pathway. Cell Biosci. 11, 163 (2021).

110. Starska, K. et al. Fibroblast growth factor receptor 1 and 3 expression is associated with regulatory PI3K/AKT kinase activity, as well as invasion and prognosis, in human laryngeal cancer. Cell Oncol. (Dordr.) 41, 253-268 (2018).

111. Wang, L., Yang, M. \& Jin, H. PI3K/AKT phosphorylation activates ERRà by upregulating PGC-1à and PGC-1á in gallbladder cancer. Mol. Med. Rep 24, 613 (2021).

112. Hou, Y., Li, H. \& Huo, W. THBS4 silencing regulates the cancer stem cell-like properties in prostate cancer via blocking the PI3K/Akt pathway. Prostate $\mathbf{8 0}$, 753-763 (2020).

113. Haddadi, N. et al. PTEN/PTENP1: 'Regulating the regulator of RTK-dependent PI3K/Akt signalling', new targets for cancer therapy. Mol. Cancer 17, 37 (2018).

114. Kim, G. W. et al. HBV-induced increased N6 methyladenosine modification of PTEN RNA affects innate immunity and contributes to HCC. Hepatology 73, 533-547 (2021).

115. Zhou, Y. et al. Hydrazinocurcumin and 5-fluorouracil enhance apoptosis and restrain tumorigenicity of HepG2 cells via disrupting the PTEN-mediated PI3K/ Akt signaling pathway. Biomed. Pharmacother. 129, 109851 (2020).

116. Nguyen Huu, T. et al. Redox regulation of PTEN by peroxiredoxins. Antioxidants 10, 302 (2021)
117. Henager, S. H., Henriquez, S., Dempsey, D. R. \& Cole, P. A. Analysis of site-specific phosphorylation of PTEN by using enzyme-catalyzed expressed protein ligation. Chembiochem 21, 64-68 (2020).

118. Li, J., Tanhehco, E. J. \& Russell, B. Actin dynamics is rapidly regulated by the PTEN and PIP2 signaling pathways leading to myocyte hypertrophy. Am. J. Physiol. Heart Circ. Physiol. 307, H1618-H1625 (2014).

119. Zhang, X. et al. IMP3 accelerates the progression of prostate cancer through inhibiting PTEN expression in a SMURF1-dependent way. J. Exp. Clin. Cancer Res. 39, 190 (2020).

120. Wang, J., Fry, C. M. E. \& Walker, C. L. Carboxyl-terminal modulator protein regulates Akt signaling during skeletal muscle atrophy in vitro and a mouse model of amyotrophic lateral sclerosis. Sci. Rep. 9, 3920 (2019).

121. Li, J., Shan, W. \& Zuo, Z. Age-related upregulation of carboxyl terminal modulator protein contributes to the decreased brain ischemic tolerance in older rats. Mol. Neurobiol. 55, 6145-6154 (2018).

122. Liu, T. et al. HEATR1 negatively regulates Akt to Help sensitize pancreatic cancer cells to chemotherapy. Cancer Res. 76, 572-581 (2016).

123. Liu, L. et al. Inhibition of protein phosphatase $2 A$ sensitizes mucoepidermoid carcinoma to chemotherapy via the PI3K-AKT pathway in response to insulin stimulus. Cell Physiol. Biochem. 50, 317-331 (2018).

124. Jeck, W. R. \& Sharpless, N. E. Detecting and characterizing circular RNAs. Nat. Biotechnol. 32, 453-461 (2014).

125. Zhang, G. et al. Identification and characterization of circular RNAs during the sea buckthorn fruit development. RNA Biol. 16, 354-361 (2019).

126. Tomar, D., Yadav, A. S., Kumar, D., Bhadauriya, G. \& Kundu, G. C. Non-coding RNAs as potential therapeutic targets in breast cancer. Biochim. Biophys. Acta Gene Regul. Mech. 1863, 194378 (2020).

127. Szabo, L. \& Salzman, J. Detecting circular RNAs: bioinformatic and experimental challenges. Nat. Rev. Genet. 17, 679-692 (2016).

128. Salzman, J. Circular RNA expression: its potential regulation and function. Trends Genet. 32, 309-316 (2016).

129. Kristensen, L. S. et al. The biogenesis, biology and characterization of circular RNAs. Nat. Rev. Genet. 20, 675-691 (2019).

130. Beermann, J., Piccoli, M. T., Viereck, J. \& Thum, T. Non-coding RNAs in development and disease: background, mechanisms, and therapeutic approaches. Physiol. Rev. 96, 1297-1325 (2016).

131. Toptan, T. et al. Circular DNA tumor viruses make circular RNAs. Proc. Natl Acad. Sci. USA 115, E8737-e8745 (2018).

132. Guarnerio, J. et al. Intragenic antagonistic roles of protein and circRNA in tumorigenesis. Cell Res. 29, 628-640 (2019).

133. Li, S. et al. Screening for functional circular RNAs using the CRISPR-Cas 13 system. Nat. Methods 18, 51-59 (2021).

134. Li, Y., Ge, Y. Z., Xu, L. \& Jia, R. Circular RNA ITCH: a novel tumor suppressor in multiple cancers. Life Sci. 254, 117176 (2020).

135. Chen, L. L. The biogenesis and emerging roles of circular RNAs. Nat. Rev. Mol. Cell Biol. 17, 205-211 (2016).

136. $\mathrm{Yu}$, J. et al. Circular RNA CSMARCA5 inhibits growth and metastasis in hepatocellular carcinoma. J. Hepatol. 68, 1214-1227 (2018).

137. Gao, X. et al. Circular RNA-encoded oncogenic E-cadherin variant promotes glioblastoma tumorigenicity through activation of EGFR-STAT3 signalling. Nat. Cell Biol. 23, 278-291 (2021).

138. Tan, S. et al. Circular RNA F-circEA produced from EML4-ALK fusion gene as a novel liquid biopsy biomarker for non-small cell lung cancer. Cell Res. 28, 693-695 (2018).

139. Goodall, G. J. \& Wickramasinghe, V. O. RNA in cancer. Nat. Rev. Cancer 21, 22-36 (2021).

140. He, J. et al. Circular RNA MAPK4 (circ-MAPK4) inhibits cell apoptosis via MAPK signaling pathway by sponging miR-125a-3p in gliomas. Mol. Cancer 19, 17 (2020).

141. Hall, I. F. et al. Circ_Lrp6, a circular RNA enriched in vascular smooth muscle cells, acts as a sponge regulating miRNA-145 function. Circ. Res. 124, 498-510 (2019).

142. Wang, R. et al. ElF4A3-induced circular RNA MMP9 (circMMP9) acts as a sponge of miR-124 and promotes glioblastoma multiforme cell tumorigenesis. Mol. Cancer 17, 166 (2018).

143. Chen, L. et al. Circular RNA 100146 functions as an oncogene through direct binding to miR-361-3p and miR-615-5p in non-small cell lung cancer. Mol. Cancer 18, 13 (2019).

144. Hill, M. \& Tran, N. MicroRNAs regulating microRNAs in cancer. Trends Cancer 4, 465-468 (2018).

145. Chen, X. et al. TDP-43 regulates cancer-associated microRNAs. Protein Cell 9 , 848-866 (2018).

146. Lee, T. J. et al. Strategies to modulate MicroRNA functions for the treatment of cancer or organ injury. Pharm. Rev. 72, 639-667 (2020). 
147. Chen, Y. et al. Circular RNA circAGO2 drives cancer progression through facilitating HuR-repressed functions of AGO2-miRNA complexes. Cell Death Differ. 26, 1346-1364 (2019).

148. Zhang, X. et al. Circular RNA circNRIP1 acts as a microRNA-149-5p sponge to promote gastric cancer progression via the AKT1/mTOR pathway. Mol. Cancer 18, 20 (2019).

149. Chen, L. Y. et al. The circular RNA circ-ERBIN promotes growth and metastasis of colorectal cancer by miR-125a-5p and miR-138-5p/4EBP-1 mediated capindependent HIF-1a translation. Mol. Cancer 19, 164 (2020).

150. Xie, F. et al. Circular RNA BCRC-3 suppresses bladder cancer proliferation through miR-182-5p/p27 axis. Mol. Cancer 17, 144 (2018).

151. Lu, Q. et al. Circular RNA circSLC8A1 acts as a sponge of miR-130b/miR-494 in suppressing bladder cancer progression via regulating PTEN. Mol. Cancer 18, 111 (2019).

152. $\mathrm{Su}, \mathrm{H}$. et al. Circular RNA cTFRC acts as the sponge of microRNA-107 to promote bladder carcinoma progression. Mol. Cancer 18, 27 (2019).

153. Zhang, Y. et al. Circular intronic long noncoding RNAs. Mol. Cell 51, 792-806 (2013).

154. Ma, N. et al. circTulp4 functions in Alzheimer's disease pathogenesis by regulating its parental gene, Tulp4. Mol. Ther. 29, 2167-2181 (2021).

155. Li, Z. et al. Exon-intron circular RNAs regulate transcription in the nucleus. Nat. Struct. Mol. Biol. 22, 256-264 (2015).

156. Zang, J., Lu, D. \& Xu, A. The interaction of circRNAs and RNA binding proteins: an important part of circRNA maintenance and function. J. Neurosci. Res 98, 87-97 (2020).

157. Wang, Z. \& Lei, X. Matrix factorization with neural network for predicting circRNA-RBP interactions. BMC Bioinforma. 21, 229 (2020).

158. Zhu, Y. J. et al. Circular RNAs negatively regulate cancer stem cells by physically binding FMRP against CCAR1 complex in hepatocellular carcinoma. Theranostics 9, 3526-3540 (2019)

159. Prats, A. C. et al. Circular RNA, the key for translation. Int. J. Mol. Sci. 21, 8591 (2020).

160. Zheng, S. L., Li, L. \& Zhang, H. P. Progress on translation ability of circular RNA. Yi Chuan 42, 423-434 (2020).

161. Li, R. et al. CircRNA: a rising star in gastric cancer. Cell Mol. Life Sci. 77, 1661-1680 (2020).

162. Verduci, L., Strano, S., Yarden, Y. \& Blandino, G. The circRNA-microRNA code: emerging implications for cancer diagnosis and treatment. Mol. Oncol. 13, 669-680 (2019)

163. Yu, T. et al. CircRNAs in cancer metabolism: a review. J. Hematol. Oncol. 12, 90 (2019).

164. Kristensen, L. S., Hansen, T. B., Venø, M. T. \& Kjems, J. Circular RNAs in cancer: opportunities and challenges in the field. Oncogene 37, 555-565 (2018).

165. Hansen, T. B., Kjems, J. \& Damgaard, C. K. Circular RNA and miR-7 in cancer. Cancer Res. 73, 5609-5612 (2013).

166. Zhan, W. et al. Circular RNA hsa circRNA 103809 promoted hepatocellular carcinoma development by regulating miR-377-3p/FGFR1/ERK axis. J. Cell Physiol. 235, 1733-1745 (2020).

167. Zhao, Z. J. \& Shen, J. Circular RNA participates in the carcinogenesis and the malignant behavior of cancer. RNA Biol. 14, 514-521 (2017).

168. Tewari, D., Patni, P., Bishayee, A., Sah, A. N. \& Bishayee, A. Natural products targeting the PI3K-Akt-mTOR signaling pathway in cancer: a novel therapeutic strategy. Semin. Cancer Biol. S1044-579X, 30405-5 (2019).

169. Alzahrani, A. S. PI3K/Akt/mTOR inhibitors in cancer: at the bench and bedside. Semin Cancer Biol. 59, 125-132 (2019).

170. O'Donnell, J. S., Massi, D., Teng, M. W. L. \& Mandala, M. PI3K-AKT-mTOR inhibition in cancer immunotherapy, redux. Semin. Cancer Biol. 48, 91-103 (2018).

171. Shi, Y. et al. Circular RNA LPAR3 sponges microRNA-198 to facilitate esophageal cancer migration, invasion, and metastasis. Cancer Sci. 111, 2824-2836 (2020).

172. He, Y. et al. CircVRK1 regulates tumor progression and radioresistance in esophageal squamous cell carcinoma by regulating miR-624-3p/PTEN/PI3K/AKT signaling pathway. Int. J. Biol. Macromol. 125, 116-123 (2019).

173. Chen, Z. et al. Circular RNA LARP4 sponges miR-1323 and hampers progression of esophageal squamous cell carcinoma through modulating PTEN/PI3K/AKT pathway. Dig. Dis. Sci. 65, 2272-2283 (2020).

174. Liu, Z. et al. Silence of CZNF292 suppresses the growth, migration, and invasion of human esophageal cancer Eca-109 cells via upregulating miR-206. J. Cell Biochem. 121, 2354-2362 (2020).

175. Song, $\mathrm{H}$. et al. CircPIP5K1A activates KRT80 and PI3K/AKT pathway to promote gastric cancer development through sponging miR-671-5p. Biomed. Pharmacother. 126, 109941 (2020).

176. Peng, Y. K. et al. Circular RNA hsa_circ 0010882 promotes the progression of gastric cancer via regulation of the PI3K/Akt/mTOR signaling pathway. Eur. Rev. Med. Pharm. Sci. 24, 1142-1151 (2020).
177. Li, J., Yang, Y., Xu, D. \& Cao, L. hsa_circ_0023409 accelerates gastric cancer cell growth and metastasis through regulating the IRS4/PI3K/AKT Pathway. Cell Transpl. 30, 963689720975390 (2021).

178. Pan, $\mathrm{H}$. et al. Overexpression of circular RNA ciRS-7 abrogates the tumor suppressive effect of miR-7 on gastric cancer via PTEN/PI3K/AKT signaling pathway. J. Cell Biochem. 119, 440-446 (2018).

179. Sun, B. et al. Circular RNA circMAN2B2 promotes growth and migration of gastric cancer cells by down-regulation of miR-145. J. Clin. Lab. Anal. 34, e23215 (2020).

180. Wang, X., Zhang, Y., Li, W. \& Liu, X. Knockdown of cir_RNA PVT1 elevates gastric cancer cisplatin sensitivity via sponging miR-152-3p. J. Surg. Res. 261, 185-195 (2021).

181. Tu, F. L. et al. Circ-0001313/miRNA-510-5p/AKT2 axis promotes the development and progression of colon cancer. Am. J. Transl. Res. 12, 281-291 (2020).

182. Cui, W., Dai, J., Ma, J. \& Gu, H. circCDYL/microRNA-105-5p participates in modulating growth and migration of colon cancer cells. Gen. Physiol. Biophys. 38, 485-495 (2019).

183. Wang, J., Luo, J., Liu, G. \& Li, X. Circular RNA hsa_circ_0008285 inhibits colorectal cancer cell proliferation and migration via the miR-382-5p/PTEN axis. Biochem. Biophys. Res. Commun. 527, 503-510 (2020).

184. Lin, Q. et al. Circular RNA circCDK13 suppresses cell proliferation, migration and invasion by modulating the JAK/STAT and PI3K/AKT pathways in liver cancer. Int. J. Oncol. 53, 246-256 (2018).

185. Fu, H. W. et al. Circ-IGF1R has pro-proliferative and anti-apoptotic effects in HCC by activating the PI3K/AKT pathway. Gene 716, 144031 (2019).

186. Yu, Q., Dai, J. \& Shu, M. Circular RNA-0072309 has antitumor influences in Hep3B cell line by targeting microRNA-665. Biofactors 46, 1618-1629 (2020).

187. Zheng, H. et al. A circular RNA hsa_circ_0079929 inhibits tumor growth in hepatocellular carcinoma. Cancer Manag. Res. 11, 443-454 (2019).

188. Sun, X. H., Wang, Y. T., Li, G. F., Zhang, N. \& Fan, L. Serum-derived three-circRNA signature as a diagnostic biomarker for hepatocellular carcinoma. Cancer Cell Int. 20, 226 (2020).

189. Tan, Y. et al. Antitumor effects of circ-EPHB4 in hepatocellular carcinoma via inhibition of HIF-1a. Mol. Carcinog. 58, 875-886 (2019).

190. Wei, Y. et al. A noncoding regulatory RNAs network driven by Circ-CDYL acts specifically in the early stages hepatocellular carcinoma. Hepatology $\mathbf{7 1}$, 130-147 (2020).

191. Eichenmüller, M. et al. The genomic landscape of hepatoblastoma and their progenies with HCC-like features. J. Hepatol. 61, 1312-1320 (2014).

192. Rougemont, A. L., McLin, V. A., Toso, C. \& Wildhaber, B. E. Adult hepatoblastoma: learning from children. J. Hepatol. 56, 1392-1403 (2012).

193. Zhen, N. et al. CircHMGCS1 promotes hepatoblastoma cell proliferation by regulating the IGF signaling pathway and glutaminolysis. Theranostics $\mathbf{9}$ 900-919 (2019).

194. Kong, Y. et al. circNFIB1 inhibits lymphangiogenesis and lymphatic metastasis via the miR-486-5p/PIK3R1/VEGF-C axis in pancreatic cancer. Mol. Cancer 19, 82 (2020).

195. Zhang, T., Li, M., Lu, H. \& Peng, T. Up-regulation of circEIF6 contributes to pancreatic cancer development through targeting miR-557/SLC7A11/PI3K/AKT signaling. Cancer Manag. Res. 13, 247-258 (2021).

196. Guo, X. et al. Circular RNA circBFAR promotes the progression of pancreatic ductal adenocarcinoma via the miR-34b-5p/MET/Akt axis. Mol. Cancer 19, 83 (2020).

197. Shi, F., Shi, Z., Zhao, Y. \& Tian, J. CircRNA hsa-circ-0014359 promotes glioma progression by regulating miR-153/PI3K signaling. Biochem. Biophys. Res. Commun. 510, 614-620 (2019).

198. $\mathrm{He}, \mathrm{Q}$. et al. MOV10 binding circ-DICER1 regulates the angiogenesis of glioma via miR-103a-3p/miR-382-5p mediated ZIC4 expression change. J. Exp. Clin. Cancer Res. 38, 9 (2019).

199. Yin, H. \& Cui, X. Knockdown of circHIPK3 facilitates temozolomide sensitivity in glioma by regulating cellular behaviors through miR-524-5p/KIF2A-mediated PI3K/AKT pathway. Cancer Biother. Radiopharm. 36, 556-567 (2021).

200. Zheng, K. et al. CircRNA PIP5K1A promotes the progression of glioma through upregulation of the TCF12/PI3K/AKT pathway by sponging miR-515-5p. Cancer Cell Int. 21, 27 (2021).

201. Chi, G., Xu, D., Zhang, B. \& Yang, F. Matrine induces apoptosis and autophagy of glioma cell line U251 by regulation of circRNA-104075/BCL-9. Chem. Biol. Interact. 308, 198-205 (2019).

202. Mutalifu, N. et al. Circ_0000215 increases the expression of CXCR2 and promoted the progression of glioma cells by sponging miR-495-3p. Technol. Cancer Res. Treat. 19, 1533033820957026 (2020).

203. Tan, A. C. et al. Management of glioblastoma: state of the art and future directions. CA Cancer J. Clin. 70, 299-312 (2020).

204. Lim, M., Xia, Y., Bettegowda, C. \& Weller, M. Current state of immunotherapy for glioblastoma. Nat. Rev. Clin. Oncol. 15, 422-442 (2018). 
205. Gan, H. K., van den Bent, M., Lassman, A. B., Reardon, D. A. \& Scott, A. M. Antibody-drug conjugates in glioblastoma therapy: the right drugs to the right cells. Nat. Rev. Clin. Oncol. 14, 695-707 (2017).

206. Xin, J., Zhang, X. Y., Sun, D. K., Tian, L. Q. \& Xu, P. Up-regulated circular RNA hsa_circ_0067934 contributes to glioblastoma progression through activating PI3K-AKT pathway. Eur. Rev. Med. Pharm. Sci. 23, 3447-3454 (2019).

207. Chi, G., Yang, F., Xu, D. \& Liu, W. Silencing hsa_circ_PVT1 (circPVT1) suppresses the growth and metastasis of glioblastoma multiforme cells by up-regulation of miR-199a-5p. Artif. Cells Nanomed. Biotechnol. 48, 188-196 (2020).

208. Cohen, M. A. et al. Formation of human neuroblastoma in mouse-human neural crest chimeras. Cell Stem Cell 26, 579-592.e576 (2020).

209. Delloye-Bourgeois, C. et al. Microenvironment-driven shift of cohesion/detachment balance within tumors induces a switch toward metastasis in neuroblastoma. Cancer Cell 32, 427-443.e428 (2017).

210. Fletcher, J. I. et al. Too many targets, not enough patients: rethinking neuroblastoma clinical trials. Nat. Rev. Cancer 18, 389-400 (2018).

211. Zhang, L. et al. Comprehensive characterization of circular RNAs in neuroblastoma cell lines. Technol. Cancer Res. Treat. 19, 1533033820957622 (2020).

212. Lecoq, A. L., Kamenický, P., Guiochon-Mantel, A. \& Chanson, P. Genetic mutations in sporadic pituitary adenomas-what to screen for? Nat. Rev. Endocrinol. 11, 43-54 (2015).

213. Elston, M. S., McDonald, K. L., Clifton-Bligh, R. J. \& Robinson, B. G. Familial pituitary tumor syndromes. Nat. Rev. Endocrinol. 5, 453-461 (2009).

214. Wang, J. et al. Circular RNA in invasive and recurrent clinical nonfunctioning pituitary adenomas: expression profiles and bioinformatic analysis. World Neurosurg. 117, e371-e386 (2018).

215. Linehan, W. M. et al. The metabolic basis of kidney cancer. Cancer Discov. 9, 1006-1021 (2019).

216. Linehan, W. M. Genetic basis of kidney cancer: role of genomics for the development of disease-based therapeutics. Genome Res. 22, 2089-2100 (2012).

217. Junker, K. et al. Potential role of genetic markers in the management of kidney cancer. Eur. Urol. 63, 333-340 (2013).

218. Chen, T., Shao, S., Li, W., Liu, Y. \& Cao, Y. The circular RNA hsa-circ-0072309 plays anti-tumour roles by sponging miR-100 through the deactivation of PI3K/AKT and mTOR pathways in the renal carcinoma cell lines. Artif. Cells Nanomed. Biotechnol. 47, 3638-3648 (2019).

219. Chen, T., Yu, Q., Xin, L. \& Guo, L. Circular RNA circC3P1 restrains kidney cancer cell activity by regulating miR-21/PTEN axis and inactivating PI3K/AKT and NFkB pathways. J. Cell Physiol. 235, 4001-4010 (2020).

220. Yao, J. et al. ZNF139/circZNF139 promotes cell proliferation, migration and invasion via activation of PI3K/AKT pathway in bladder cancer. Aging (Albany NY) 12, 9915-9934 (2020).

221. Siegel, D. A., O'Neil, M. E., Richards, T. B., Dowling, N. F. \& Weir, H. K. Prostate cancer incidence and survival, by stage and race/ethnicity-United States, 20012017. MMWR Morb. Mortal. Wkly Rep. 69, 1473-1480 (2020).

222. Gandaglia, G. et al. Structured population-based prostate-specific antigen screening for prostate cancer: the European Association of Urology Position in 2019. Eur. Urol. 76, 142-150 (2019).

223. Zhang, L. et al. The first integrins $\beta 3$-mediated cellular and nuclear targeting therapeutics for prostate cancer. Biomaterials 223, 119471 (2019).

224. Wong, M. C. et al. Global incidence and mortality for prostate cancer: analysis of temporal patterns and trends in 36 countries. Eur. Urol. 70, 862-874 (2016).

225. Chen, W. et al. Circular RNA CircNOLC1, upregulated by NF-KappaB, promotes the progression of prostate cancer via miR-647/PAQR4 axis. Front. Cell Dev. Biol. 8, 624764 (2020).

226. Li, S. et al. Circular RNA cir-ITCH is a potential therapeutic target for the treatment of castration-resistant prostate cancer. Biomed. Res. Int. 2020, 7586521 (2020).

227. Shi, J. et al. Circular RNA circMBOAT2 promotes prostate cancer progression via a miR-1271-5p/mTOR axis. Aging (Albany NY) 12, 13255-13280 (2020).

228. Yan, Z., Xiao, Y., Chen, Y. \& Luo, G. Screening and identification of epithelial-tomesenchymal transition-related circRNA and miRNA in prostate cancer. Pathol. Res. Pr. 216, 152784 (2020).

229. Ventriglia, J. et al. Immunotherapy in ovarian, endometrial and cervical cancer: state of the art and future perspectives. Cancer Treat. Rev. 59, 109-116 (2017).

230. Mu, A. K., Lim, B. K., Aminudin, N., Hashim, O. H. \& Shuib, A. S. Application of SELDI-TOF in N-glycopeptides profiling of the urine from patients with endometrial, ovarian and cervical cancer. Arch. Physiol. Biochem. 122, 111-116 (2016).

231. Yalan, S., Yanfang, L., He, C., Yujie, T. Circular RNA circRHOBTB3 inhibits ovarian cancer progression through PI3K/AKT signaling pathway. Panminerva Med. Preprint at http://www.medrxiv.org/content/ https://doi.org/10.23736/S00310808.20.03957-9 (2020)

232. Wang, Y., Yin, L. \& Sun, X. CircRNA hsa circ 0002577 accelerates endometrial cancer progression through activating IGF1R/PI3K/Akt pathway. J. Exp. Clin. Cancer Res. 39, 169 (2020).
233. Yang, W. \& Xie, T. Hsa_circ_CSPP1/MiR-361-5p/ITGB1 regulates proliferation and migration of cervical cancer (CC) by modulating the PI3K-Akt signaling pathway. Reprod. Sci. 27, 132-144 (2020).

234. Kim, J., Gosnell, J. E. \& Roman, S. A. Geographic influences in the global rise of thyroid cancer. Nat. Rev. Endocrinol. 16, 17-29 (2020).

235. Ito, Y., Nikiforov, Y. E., Schlumberger, M. \& Vigneri, R. Increasing incidence of thyroid cancer: controversies explored. Nat. Rev. Endocrinol. 9, 178-184 (2013).

236. Lin, R. Y. Thyroid cancer stem cells. Nat. Rev. Endocrinol. 7, 609-616 (2011).

237. Mazeh, H. \& Chen, H. Advances in surgical therapy for thyroid cancer. Nat. Rev. Endocrinol. 7, 581-588 (2011).

238. Wang, H., Yan, X., Zhang, H. \& Zhan, X. CircRNA circ_0067934 overexpression correlates with poor prognosis and promotes thyroid carcinoma progression. Med. Sci. Monit. 25, 1342-1349 (2019).

239. Long, M. Y. et al. Comprehensive circular RNA profiling reveals the regulatory role of circRNA_0007694 in papillary thyroid carcinoma. Am. J. Transl. Res. 12, 1362-1378 (2020).

240. Li, Z. et al. Circ_PSD3 promotes the progression of papillary thyroid carcinoma via the miR-637/HEMGN axis. Life Sci. 264, 118622 (2021).

241. Jonas, D. E. et al. Screening for lung cancer with low-dose computed tomography: updated evidence report and systematic review for the US preventive services task force. J. Am. Med. Assoc. USA 325, 971-987 (2021).

242. Duruisseaux, M. \& Esteller, M. Lung cancer epigenetics: from knowledge to applications. Semin. Cancer Biol. 51, 116-128 (2018).

243. Gridelli, C. et al. Non-small-cell lung cancer. Nat. Rev. Dis. Prim. 1, 15009 (2015).

244. Oser, M. G., Niederst, M. J., Sequist, L. V. \& Engelman, J. A. Transformation from non-small-cell lung cancer to small-cell lung cancer: molecular drivers and cells of origin. Lancet Oncol. 16, e165-e172 (2015).

245. Yao, J., Xu, G., Zhu, L. \& Zheng, H. circGFRA1 enhances NSCLC progression by sponging miR-188-3p. Onco Targets Ther. 13, 549-558 (2020).

246. Xu, X., Zhou, X., Gao, C. \& Cui, Y. Hsa_circ_0018818 knockdown suppresses tumorigenesis in non-small cell lung cancer by sponging miR-767-3p. Aging (Albany NY) 12, 7774-7785 (2020).

247. Song, J., Shi, W., Gao, Z., Liu, X. \& Wang, W. Downregulation of circRNA_100876 inhibited progression of NSCLC in vitro via targeting miR-636. Technol. Cancer Res. Treat. 19, 1533033820951817 (2020).

248. Isakoff, M. S., Bielack, S. S., Meltzer, P. \& Gorlick, R. Osteosarcoma: current treatment and a collaborative pathway to success. J. Clin. Oncol. 33, 3029-3035 (2015).

249. Angulo, P. et al. Natural compounds targeting major cell signaling pathways: a novel paradigm for osteosarcoma therapy. J. Hematol. Oncol. 10, 10 (2017).

250. Li, S. et al. Circular RNA 0001785 regulates the pathogenesis of osteosarcoma as a ceRNA by sponging miR-1200 to upregulate HOXB2. Cell Cycle 18, 1281-1291 (2019).

251. Lin, E., Liu, S., Xiang, W., Zhang, H. \& Xie, C. CircEIF4G2 promotes tumorigenesis and progression of osteosarcoma by sponging miR-218. Biomed. Res Int 2020, 8386936 (2020).

252. Ren, C. et al. The circular RNA circ-ITCH acts as a tumour suppressor in osteosarcoma via regulating miR-22. Artif. Cells Nanomed. Biotechnol. 47, 3359-3367 (2019).

253. Zhang, C., Na, N., Liu, L. \& Qiu, Y. CircRNA hsa circ 0005909 promotes cell proliferation of osteosarcoma cells by targeting miR-338-3p/HMGA1 Axis. Cancer Manag. Res 13, 795-803 (2021).

254. Omori, $\mathrm{H}$. et al. YAP1 is a potent driver of the onset and progression of oral squamous cell carcinoma. Sci. Adv. 6, eaay3324 (2020)

255. Peng, Q. S. et al. circRNA_0000140 suppresses oral squamous cell carcinoma growth and metastasis by targeting miR-31 to inhibit Hippo signaling pathway. Cell Death Dis. 11, 112 (2020).

256. Pai, S. et al. CD47-SIRPa signaling induces epithelial-mesenchymal transition and cancer stemness and links to a poor prognosis in patients with oral squamous cell carcinoma. Cells 8, 1658 (2019).

257. Deng, W. et al. Microarray profile of circular RNAs identifies hsa_circRNA 102459 and hsa_circRNA_043621 as important regulators in oral squamous cell carcinoma. Oncol. Rep. 42, 2738-2749 (2019).

258. Morgan, G. J., Walker, B. A. \& Davies, F. E. The genetic architecture of multiple myeloma. Nat. Rev. Cancer 12, 335-348 (2012).

259. Ledergor, G. et al. Single cell dissection of plasma cell heterogeneity in symptomatic and asymptomatic myeloma. Nat. Med. 24, 1867-1876 (2018).

260. Kumar, S. K. et al. Multiple myeloma. Nat. Rev. Dis. Prim. 3, 17046 (2017).

261. Wang, Y., Lin, Q., Song, C., Ma, R. \& Li, X. Circ_0007841 promotes the progression of multiple myeloma through targeting miR-338-3p/BRD4 signaling cascade. Cancer Cell Int. 20, 383 (2020).

262. Qiu, X., Wang, Q., Song, H., Shao, D. \& Xue, J. circ 103809 promotes breast cancer progression by regulating the PI3K/AKT signaling pathway. Oncol. Lett. 19, 3725-3730 (2020). 
263. Wu, D., Jia, H., Zhang, Z. \& Li, S. Circ-PRMT5 promotes breast cancer by the miR509-3p/TCF7L2 axis activating the PI3K/AKT pathway. J. Gene Med. 23, e3300 (2021).

264. Chen, Z. G. et al. Circular RNA CirCHIPK3 promotes cell proliferation and invasion of breast cancer by sponging miR-193a/HMGB1/PI3K/AKT axis. Thorac. Cancer 11, 2660-2671 (2020)

265. Zhang, X. Y. \& Mao, L. Circular RNA Circ_0000442 acts as a sponge of MiR-148b$3 p$ to suppress breast cancer via PTEN/PI3K/Akt signaling pathway. Gene $\mathbf{7 6 6}$ $145113(2021)$

266. Xu, J. H., Wang, Y. \& Xu, D. Hsa_circ_001569 is an unfavorable prognostic factor and promotes cell proliferation and metastasis by modulating PI3K-AKT pathway in breast cancer. Cancer Biomark. 25, 193-201 (2019).

267. Li, H. et al. Hsa_circ_0000199 facilitates chemo-tolerance of triple-negative breast cancer by interfering with miR-206/613-led PI3K/Akt/mTOR signaling. Aging (Albany NY) 13, 4522-4551 (2021).

268. Redman, M. W. et al. Biomarker-driven therapies for previously treated squamous non-small-cell lung cancer (Lung-MAP SWOG S1400): a biomarker-driven master protocol. Lancet Oncol. 21, 1589-1601 (2020).

269. Sveen, A., Kopetz, S. \& Lothe, R. A. Biomarker-guided therapy for colorectal cancer: strength in complexity. Nat. Rev. Clin. Oncol. 17, 11-32 (2020).

270. Whiteside, T. L. Validation of plasma-derived small extracellular vesicles as cancer biomarkers. Nat. Rev. Clin. Oncol. 17, 719-720 (2020).

271. Rebbeck, T. R. et al. Precision prevention and early detection of cancer: fundamental principles. Cancer Discov. 8, 803-811 (2018).

272. Wender, R. C., Brawley, O. W., Fedewa, S. A., Gansler, T. \& Smith, R. A. A blueprint for cancer screening and early detection: advancing screening's contribution to cancer control. CA Cancer J. Clin. 69, 50-79 (2019).

273. Borrebaeck, C. A. Precision diagnostics: moving towards protein biomarker signatures of clinical utility in cancer. Nat. Rev. Cancer 17, 199-204 (2017).

274. Lin, X. J. et al. A serum microRNA classifier for early detection of hepatocellular carcinoma: a multicentre, retrospective, longitudinal biomarker identification study with a nested case-control study. Lancet Oncol. 16, 804-815 (2015).

275. Wu, L. \& Qu, X. Cancer biomarker detection: recent achievements and challenges. Chem. Soc. Rev. 44, 2963-2997 (2015).
276. Fan, L., Cao, Q., Liu, J., Zhang, J. \& Li, B. Circular RNA profiling and its potential for esophageal squamous cell cancer diagnosis and prognosis. Mol. Cancer 18, 16 (2019).

277. Ding, L. et al. Circular RNA circ-DONSON facilitates gastric cancer growth and invasion via NURF complex dependent activation of transcription factor SOX4. Mol. Cancer 18, 45 (2019).

278. Cooper, B. M., legre, J., Donovan, Daniel H. O'., Ölwegård Halvarsson, M. \& Spring, D. R. Peptides as a platform for targeted therapeutics for cancer: peptide-drug conjugates (PDCs). Chem. Soc. Rev. 50, 1480-1494 (2021).

279. Oh, D. Y. \& Bang, Y. J. HER2-targeted therapies-a role beyond breast cancer. Nat. Rev. Clin. Oncol. 17, 33-48 (2020).

280. Comoglio, P. M., Trusolino, L. \& Boccaccio, C. Known and novel roles of the MET oncogene in cancer: a coherent approach to targeted therapy. Nat. Rev. Cancer 18, 341-358 (2018).

281. Srinivasarao, M., Galliford, C. V. \& Low, P. S. Principles in the design of ligandtargeted cancer therapeutics and imaging agents. Nat. Rev. Drug Discov. 14, 203-219 (2015).

282. Schmitt, M. W., Loeb, L. A. \& Salk, J. J. The influence of subclonal resistance mutations on targeted cancer therapy. Nat. Rev. Clin. Oncol. 13, 335-347 (2016).

(i) Open Access This article is licensed under a Creative Commons c. Attribution 4.0 International License, which permits use, sharing, adaptation, distribution and reproduction in any medium or format, as long as you give appropriate credit to the original author(s) and the source, provide a link to the Creative Commons license, and indicate if changes were made. The images or other third party material in this article are included in the article's Creative Commons license, unless indicated otherwise in a credit line to the material. If material is not included in the article's Creative Commons license and your intended use is not permitted by statutory regulation or exceeds the permitted use, you will need to obtain permission directly from the copyright holder. To view a copy of this license, visit http://creativecommons. org/licenses/by/4.0/.

(c) The Author(s) 2021 